\title{
Testing for breaks in the weighting matrix
}

\author{
Ana Angulo $^{a}$, Peter Burridge ${ }^{b}$ and Jesús Mur ${ }^{a}$ \\ ${ }^{a}$ Department of Economic Analisis, University of Zaragoza, Spain \\ ${ }^{b}$ Department of Economic and Related Studies, University of York, UK
}

July 14, 2016

\begin{abstract}
The weighting matrix is a key element in the specification of a spatial model. Typically, this matrix is fixed a priori by the researcher, which is not always satisfactory. Theoretical justification for the chosen matrix tends to be very vague, and the selection problem is seldom reconsidered. However, several recent proposals advocate a more data-driven approach. In fact, if we have panel data, the weighting matrix can be estimated from the data; this facilitates the development of statistical procedures for testing various hypotheses of interest. In the paper, we focus on the assumption of stability, through time, of this matrix by adapting a collection of covariance matrix stability tests, developed in a multivariate context. The tests are compared in a Monte Carlo; two examples illustrate the proposal.
\end{abstract}

Keywords: Weights matrix; Estimation of W; Structural breaks; Tests of equality.

JEL classification: C4, C5, R1.

\footnotetext{
${ }^{*}$ Corresponding author: Jesus Mur. Department of Economic Analysis. University of Zaragoza (email: jmur@unizar.es)
} 


\section{Introduction}

Brun et al. (2005) draw attention to an interesting fact: most of the literature on gravity models finds estimated distance coefficients that increase, rather than decrease, through time. This is unexpected because (p. 103) "the common perception of globalization is that distance should be becoming less important for international trade, implying decreasing rather than increasing values of the estimated coefficient of distance". The puzzle is solved after correcting some mis-specifications in the gravity equation. Then the authors (p. 117) announce the death of distance, estimating an "11\% decrease in the impact of distance on bilateral trade over the 35-year period 1962-1996". This is just one example, that makes clear that spatial relations are not static but evolve over time. Not only may the strength of estimated spatial dependence change, but also the pattern of such dependence, often represented by a matrix, W. Another example is the model for household demand of Case (1991), where the weights in the $\mathbf{W}$ matrix depend on incomes of the different districts (so-called income distance), which means weights (i) may be endogeneous and (ii) may change continuously.

The case of an endogenous $\mathbf{W}$ matrix is well-known, though technically difficult. Only recently have Kelejian and Piras (2014) and Qu and Lee (2015) formalized its treatment using algorithms (such as generalized method of moments, GMM, or instrumental variables, IV) with good properties. Ahrens and Bhattacharjee (2015) develop a two-step Lasso algorithm to estimate the weights in a spatial autoregressive panel data model. The Lasso approach allows the authors to circumvent at the same time the problems of endogeneity, associated with the spatial lags of the endogenous variable, and of high dimensionality of the equation. Let us note that they treat the weights of $\mathbf{W}$ as unknown coefficients to be estimated so sparseness of this matrix is a key assumption (more sparseness, fewer parameters to estimate, faster convergence).

We are aware of few papers that deal with changes in the $\mathbf{W}$ matrix across time. Druska and Horrace (2004) in their study of Javan rice farm efficiency, may be the first to work formally with a time-varying $\mathbf{W}$; they use a two seasons model which involves two different pre-specified weight matrices, one for the dry season, the other for the wet season. Obviously, season changes are known in advance, and so are exogenous. Lee and $\mathrm{Yu}$ (2012) develop a quasi-maximum likelihood estimation, QML, of dynamic panel data models where spatial weights matrices are also time-varying; they are assumed 
exogeneous and known. QML estimates are consistent and asymptotically normal when both the number of spatial units and time periods increase. Moreover, Lee and $\mathrm{Yu}$ also detect significant biases in the case of substantial misspecification, with a time invariant weighting matrix assumed when the true process has time varying matrices. The magnitude of the bias increases for the estimates of the marginal direct/indirect effects. Angulo et al. (2016) focus on the problem of testing for changes in the matrix across time, building the weights by means of exponential and inverse distance decay functions; a likelihood ratio test with good properties, even for very small samples, is obtained. Our goal is along the same lines, trying to provide tools to detect changes in the weighting matrix.

The discussion below is confined to the most common situation in applied work, where the weighting matrix is exogenous (probably depending on physical distance or some other measure of separation). One of the main difficulties for testing hypotheses in relation to $\mathbf{W}$ is the potential problem of high-dimensionality given that, usually, the cross sectional dimension is larger than the temporal dimension of the data. This prevents the use of standard approaches such as maximum-likelihood, which is badly equipped to deal with singularity of the covariance matrix. Another difficulty is the lack of identification that affects most spatial models, in the sense that is difficult to separate the impact of a spatial coefficient from the effect of the W matrix to which it is applied. However, given the unambiguous relation that exists between the weighting matrix and the covariance matrix, the literature devoted to the second appears to offer a reasonable framework for the problem. We review this strand of literature looking for tests that would allow us to test for the constancy, through time, of the weights matrix. The aim is to demonstrate the usefulness of these procedures in applied research, either using a Spatial Error Model, SEM, or a Spatial Lag Model, SLM.

The second section discusses briefly the role of the $\mathbf{W}$ matrix in a typical spatial model. In Section 3 we review the recent literature devoted to the problem of comparing covariance matrices estimated using different samples. We select a set of equality tests, apparently with good properties, that address the problem. Section 4 presents the results of a Monte Carlo experiment comparing these tests. Section 5 illustrates our proposal with two case studies in which the assumption of constancy in $\mathbf{W}$ plays a crucial role. Section 6 concludes. 


\section{The $\mathrm{W}$ issue: some stylized facts}

Corrado and Fingleton (2012) pose three questions in relation to W. For what is it used? How is it built? Finally, do we need it?

The answer to the first question is simple: spatial lags of some variables are necessary because their spatial spillovers are unobservable events in real life but we have to account for their effects in our models. So, we need to speculate about their appropriate form by using proxy variables.

A matrix of weights constitutes a reasonable procedure, though it is not the only solution. Oud and Folmer (2008), for example, introduce the latent variables approach to account for spatial dependence using a structural equations model; spatially lagged variables are represented by latent variables which are measured through a set of proxies. Paci and Usai (2009) extend the use of proxy variables to the problem of measuring unobserved spatial (knowledge) flows produced over space, where geography constitutes an additional source for proxy variables. The HAC method of Kelejian and Prucha (2007) can be seen as another way of dealing with unobserved effects related to the spatial structure.

However, these cases are the exception; in fact, there are not many alternatives to the use of $\mathbf{W}$, which must therefore be defined. Roughly speaking, we may distinguish two approaches to the building of $\mathbf{W}$ (Harris et al., 2011): (i) specifying $\mathbf{W}$ exogenously; (ii) estimating $\mathbf{W}$ from data. The exogenous approach is by far the most common and includes, for example, use of a binary contiguity criterion, k-nearest neighbours, kernel functions based on distance, etc.

The second approach uses the topology of the space and the nature of the data, and takes many forms. Most are ad-hoc procedures in which an objective is selected in advance which guides the search. Kooijman (1976) was one of the first to tackle explicitly the question of estimating a $\mathbf{W}$ matrix. His suggestion, to build the weights so as to maximize the value of Moran's I, is intuitive, but is difficult to implement because of the high number of unknowns. Kooijman's suggestion is related with other more recent proposals such as Griffith (1996), who tries to find a W able to absorb the spatial effects from the data. Fernández et al. (2009) propose a specification of $\mathbf{W}$ based on a measure of entropy, the GME approach, while Mur and Paelinck (2010) focus on the maximization of the Complete Correlation Coefficient, the CCC approach. The LSM (local statistical model) of Getis and Aldstadt (2004) is very popular, as is the AMOEBA algorithm of Aldstadt and Getis (2006). 
Also included is the most recent work in which $\mathbf{W}$ is directly estimated from the data. For example, Benjanuvatra and Burridge (2015) present a QML algorithm to estimate the weights in $\mathbf{W}$ using a single cross-section, under the assumption that these weights are a function of the distance between the locations, known up to a parameter that may be estimated.

More flexible approaches to $\mathbf{W}$ are possible if repeated information about the interactions is available. The initial suggestion of Meen (1996) is to set the problem in a multivariate framework: (i) a SUR model is estimated where each equation corresponds to a region (assuming necessary homogeneity and aggregation restrictions), then (ii) spatial error dependence, if it exists, will be captured by the covariance matrix of the SUR residuals. As indicated by Meen (1996, p 360), "the advantage is that there is no need to specify in advance the form of the spatial error dependence through a weights matrix". He goes a step further suggesting a LS regression of the SUR residuals in each region on the residuals of the other regions:

$$
\hat{u}_{r t}=\sum_{\substack{j=1 \\ j \neq r}}^{n} \omega_{r j} \hat{u}_{j t}+\varepsilon_{r t}, \quad t=1, \ldots, T .
$$

Then the t-ratios can be used to assess if the sequence of weights, $\omega_{r j}$, is statistically significant in each equation. Unfortunately, there are strong endogeneity problems in (1).

Battacharjee and Jensen-Butler (2013) consider a panel data model with SEM errors:

$$
\begin{aligned}
\mathbf{y}_{t} & =\mathbf{x}_{t} \boldsymbol{\beta}+\mathbf{u}_{t} \\
\mathbf{u}_{t} & =\mathbf{R}_{n} \mathbf{W}_{n} \mathbf{u}_{t}+\boldsymbol{\varepsilon}_{t}, \quad t=1,2, \ldots, T \\
E\left\{\boldsymbol{\varepsilon}_{t}\right\} & =\mathbf{0}, \quad \operatorname{Var}\left\{\boldsymbol{\varepsilon}_{t}\right\}=\mathbf{\Sigma}_{n}=\operatorname{diag}\left\{\sigma_{1}^{2}, \sigma_{2}^{2}, \ldots, \sigma_{n}^{2}\right\} \\
E\left\{\varepsilon_{t} \varepsilon_{s}^{\prime}\right\} & =\mathbf{0}, \quad t \neq s .
\end{aligned}
$$

Here, $\mathbf{y}_{t}$ is an $(n \times 1)$ vector of observations of the endogenous variable and $\mathbf{x}_{t}$ an $(n \times k)$ matrix of exogenous regressors. $\mathbf{R}_{n}$ is an $(n \times n)$ diagonal matrix of spatial dependence coefficients $\left\{\rho_{i}, i=1, \ldots, n\right\}$ and $\mathbf{W}_{n}$ the unknown weight matrix with zeroes in its main diagonal; the nonsingularity condition, $\left|\mathbf{I}-\mathbf{R}_{n} \mathbf{W}_{n}\right| \neq 0$ applies. Moreover, $n$ is fixed and $T$ is allowed to increase. Like Meen (1996), they suggest using a consistent estimator of the covariance 
matrix of the error terms obtained from the LS residuals, $\hat{\boldsymbol{\Gamma}}=T^{-1} \sum_{t=1}^{T} \hat{\mathbf{u}}_{t} \hat{\mathbf{u}}_{t}^{\prime}$, in order to produce unique consistent estimates of matrices $\mathbf{R}_{n}, \boldsymbol{\Sigma}_{n}$ and $\mathbf{W}_{n}$, via the relation, $\hat{\boldsymbol{\Gamma}}_{n}=\hat{\mathbf{A}}_{n} \hat{\boldsymbol{\Sigma}}_{n} \hat{\mathbf{A}}_{n}^{\prime}$, where $\hat{\mathbf{A}}_{n}=\left(\mathbf{I}_{n}-\hat{\mathbf{R}}_{n} \hat{\mathbf{W}}_{n}\right)^{-1}$. There are identification problems in (2) solved by assuming that (i) the spatial autoregressive parameter is the same for all regions, so that $\mathbf{R}_{n} \mathbf{W}_{n}=\rho \mathbf{W}_{n}$ and (ii) the spatial weights matrix is symmetric.

These two conditions are also essential in the proposal of Beenstock and Felsenstein (2012) for estimating $\mathbf{W}_{n}$ in a pure panel data SLM model with unobserved random effects:

$$
\mathbf{y}_{t}=\boldsymbol{\eta}_{n}+\mathbf{R}_{n} \mathbf{W}_{n} \mathbf{y}_{t}+\varepsilon_{t}
$$

$\boldsymbol{\eta}_{n}$ being an $(n \times 1)$ vector of unobserved random effects assumed orthogonal to the idiosyncratic error terms, $\boldsymbol{\varepsilon}_{t}$.

\section{The Covariance and the Weighting matrix}

As is so often the case, tests for the constancy of effects go hand-in-hand with the development of estimation methods. Bhattacharjee and JensenButler (2013) extend the identity test of Ledoit and Wolf (2002), LW, to the case of testing for a given driver of spatial diffusion; specifically, their hypothesis is that:

$$
H_{0}: \mathbf{W}=\mathbf{W}_{0} \quad \text { vs } \quad H_{A}: \mathbf{W} \neq \mathbf{W}_{0} .
$$

For the case of model (2) the test is constructed by comparing two estimated covariance matrices. The first is a restricted estimator, efficient only under the null hypothesis while it is biased under the alternative:

$$
\hat{\boldsymbol{\Gamma}}_{W_{0}}=\left(\mathbf{I}-\hat{\mathbf{R}}_{n, r} \mathbf{W}_{0}\right)^{\prime-1} \hat{\boldsymbol{\Sigma}}_{n, r}\left(\mathbf{I}-\hat{\mathbf{R}}_{n, r} \mathbf{W}_{0}\right)^{-1} .
$$

$\hat{\mathbf{R}}_{n, r}$ and $\hat{\boldsymbol{\Sigma}}_{n, r}$ are the corresponding restricted estimates. The second, unrestricted estimator is consistent under both null and alternative, but inefficient under the null.

$$
\hat{\boldsymbol{\Gamma}}=\left(\mathbf{I}-\hat{\mathbf{R}}_{n, u} \hat{\mathbf{W}}_{n}\right)^{\prime-1} \hat{\boldsymbol{\Sigma}}_{n, u}\left(\mathbf{I}-\hat{\mathbf{R}}_{n, u} \hat{\mathbf{W}}_{n}\right)^{-1} .
$$

$\hat{\mathbf{R}}_{n, u}, \hat{\boldsymbol{\Sigma}}_{n, u}$ and $\hat{\mathbf{W}}_{n}$ are the unrestricted estimates of $\mathbf{R}, \boldsymbol{\Sigma}$ and $\mathbf{W}$. 
The LW test is consistent (under $T \rightarrow \infty$ ) and has good behaviour for samples of small to moderate size, even for the more difficult case of highdimensionality $(n>T)$; see Ledoit and Wolf (2002) for the details. Unfortunately, the LW test is not adequate to test for breaks in the weighting matrix because $\mathbf{W}_{0}$ should be specified in advance.

In line with most previous studies, our suggestion is to test for the existence of a breakpoint in $\mathbf{W}$ by using consistent estimates of the corresponding covariance matrix, $\boldsymbol{\Gamma}$. Let us assume, for the moment, that we know the data generating process, i.e. a static spatial model

$$
S_{y}\left(\rho_{y} ; \mathbf{W}_{y}\right) \mathbf{y}_{t}=\boldsymbol{\mu}+S_{x}\left(\rho_{x} ; \mathbf{W}_{x}\right) \mathbf{x}_{t} \boldsymbol{\beta}+S_{u}\left(\rho_{u} ; \mathbf{W}_{u}\right) \mathbf{u}_{t} ; \quad t=1,2,3, \ldots T
$$

where $\mathbf{y}_{t}$ and $\mathbf{u}_{t}$ are $(n \times 1)$ vectors and $\mathbf{x}_{t}$ a $(n \times k)$ matrix of $k$ covariates, $\mathbf{x}_{t}=\left(x_{1 t}, x_{2 t}, \ldots, x_{k t}\right) ; \boldsymbol{\beta}$ is a vector of $k$ parameters and $\boldsymbol{\mu}$ a $(n \times 1)$ vector of unobserved individual effects $\left(\boldsymbol{\mu} \sim\right.$ i.i.d. $\left.\left(\boldsymbol{\mu}_{0}, \boldsymbol{\Sigma}_{\mu}\right)\right)$. The terms in vector $\mathbf{u}_{t}$, are spatially and serially uncorrelated, centered on zero and have a diagonal covariance matrix, $\boldsymbol{\Lambda}$, that is constant through time. Each of the $k$ covariates, $x_{i}, i=1,2, . ., k$, conform to a stationary process with mean vector $\boldsymbol{\eta}_{x_{i}}$ and covariance matrix $\boldsymbol{\Sigma}_{x_{i}}$. Moreover, $S_{g}\left(\rho_{g} ; \mathbf{W}_{g}\right), S_{g}$ in short, refers to the spatial structure related to $g$, where $\rho_{g}$ is a spatial dependence parameter and $\mathbf{W}_{g}$ an $(n \times n)$ matrix. This structure can be a spatial moving average, an autoregressive process or another spatial mechanism that depends on a spatial driver. The weighting matrices may coincide $\left(\mathbf{W}_{g}=\mathbf{W}, \forall g\right)$, as usual in applied work, or they may be different. Let us assume that the covariates, unobserved effects and error terms are all independent, $\operatorname{Cov}\left(\boldsymbol{\mu}_{t} ; x_{i t}\right)=\operatorname{Cov}\left(\boldsymbol{\mu}_{t} ; u_{t}\right)=\operatorname{Cov}\left(\mathbf{u}_{t} ; x_{i t}\right)=\mathbf{0} ; i=1,2, . ., k ; \forall t$. Under these circunstances, the covariance matrix of vector $\mathbf{y}$ is

$$
V\left(\mathbf{y}_{t}\right)=\boldsymbol{\Gamma}=S_{y}^{-1}\left[\Sigma_{\mu}+S_{x}\left(\sum_{i=1}^{k} \beta_{i}^{2} \boldsymbol{\Sigma}_{x_{i}}\right) S_{x}^{\prime}+S_{u} \boldsymbol{\Lambda} S_{u}^{\prime}\right] S_{y}^{\prime-1} ; \forall t
$$

Our focus is on the weights matrix. It is clear that, assuming stability in the other elements, we should observe changes in $\Gamma$ if $\mathbf{W}$ itself also changes. The contrary does not apply given that the covariance matrix depends on many other terms (variances, slope coefficients, etc.). This is a limitation of the procedure. 
Assume that a sequence of $\{t=1,2, \ldots, T\}$ time series observations is available for vector $\mathbf{y}_{t}$. The null hypothesis states that the covariance matrix, $\Gamma$, is the same for the whole period, whereas the alternative introduces a breakpoint in period $T_{b}, 1<T_{b}<T$. In that case, the sample should be divided into two different samples $\left\{t=1, \ldots, T_{b}\right\}$ and $\left\{t=T_{b}+1, \ldots, T\right\}$, because different weights matrices apply in each period, $\mathbf{W}_{1}$ and $\mathbf{W}_{2}$, which in turn produce different covariance matrices, $\boldsymbol{\Gamma}_{1}$ and $\boldsymbol{\Gamma}_{2}$. We want to test if there is a break in $T_{1}$.

$$
H_{0}: \Gamma_{1}=\Gamma_{2}=\Gamma \quad \text { vs } \quad H_{A}: \Gamma_{1} \neq \Gamma_{2} .
$$

The likelihood ratio test (Anderson, 2003), LR, is one of the most popular tests in this field, for which we need (i) the assumption of a correct, known specification $^{1}$, (ii) the assumption of normality and (iii) knowledge about the breakpoints. The LR for the null hypothesis of (9) does not depend on the first order moments:

$$
L R=-2 \ln \frac{|\widehat{\boldsymbol{\Gamma}}|^{-T / 2}}{\left|\widehat{\boldsymbol{\Gamma}}_{1}\right|^{-T_{1} / 2}\left|\widehat{\boldsymbol{\Gamma}}_{2}\right|^{-T_{2} / 2}} \rightarrow \chi^{2}\left(\frac{n(n+1)}{2}\right)
$$

as $T \rightarrow \infty$ for $n$ fixed.

$\widehat{\boldsymbol{\Gamma}}$ and $\widehat{\boldsymbol{\Gamma}}_{i} ; i=1,2$ are the sample covariance matrices under the null and alternative hypotheses. The statistic of (10) is easily extended to the case of $d$ known breakpoints. The LR is severely affected by the highdimensionality problem which means that: (i)- the $L R$ test degenerates when $n \geqslant T_{i}, i=1,2$, because the sample covariance matrices are singular; (ii)if both dimensions, $T$ and $n$, are large $\left(T, n \rightarrow \infty\right.$, but $\left.n<T_{i}\right)$ the percentage of false rejections increases dramatically. Ledoit and Wolf (2002) refer to the ratio $\frac{n}{T_{i}}$ as the concentration $c_{i}$ index, which should be considerably lower than 1 (according to their simulation results, less than 0.05). Bai et al. (2009) later on show that the $L R$ statistic drifts to infinity almost surely, given that $n \rightarrow \infty$, independently of the concentration $c_{i}$.

\footnotetext{
${ }^{1}$ Actually this assumption is not strictly necessary. The LR test, as well as the following tests, is obtained from a covariance matrix which can be the residual covariance matrix or the covariance matrix of $\mathbf{y}$. According to our experience, knowledge about the process improves power/size of the tests.
} 
This observation is important because spatial data usually involve a large $n$ and a finite T. As a remedy to the degeneracy problem, Schott (2007) introduces a Wald test, which only requires the covariance matrix to be nonsingular under the null (that is $T>n$ ).

Bai et al. (2009) obtain the corrections needed for the LR statistics, in mean and variance, to behave properly for the case where $(T, n \rightarrow \infty)$ with concentration $c_{i}$ indices lower than 1 in both subsamples. They show that if variables are i.i.d., under the null hypothesis the LR ratio of (10), corrected by a quantity proportional to the dimension of the matrices, asymptotically converges to a Normal distribution with finite first and second order moments; that is:

$$
T_{N}=\frac{\left(L R-n F_{T_{1} ; T_{2}}\right)-m(f)}{v(f)^{1 / 2}} \rightarrow N(0,1)
$$

where $n F_{\alpha_{1} ; \alpha_{2}}$ is the Marchenko-Pastur law of indices $\alpha_{1}$ and $\alpha_{2} ; m(f)$ and $v(f)$ are the mean and variance, respectively, of the normal random variable $\left(L R-n F_{T_{1} ; T_{2}}\right)$; see Theorem 4.1 and expressions (4.2)-(4.7) in Bai et al (2009). Note that the result of (11) applies for general i.i.d. distributions, having a fourth order moment; the $T_{N}$ test can be considered as a generalized pseudo-likelihood ratio test for non-Gaussian data.

In another strand of literature, Schott (2007), Srivastava (2007), Srivastava and Yanagihara (2010), Li and Chen (2012) and Srivastava et al. (2014) use the squared Frobenius norm to measure the distance between the null and the alternative hypotheses. The resulting statistics rely on estimates of the distance between the two covariance matrices:

$$
\operatorname{tr}\left(\boldsymbol{\Gamma}_{1}-\boldsymbol{\Gamma}_{2}\right)^{2}=\operatorname{tr} \boldsymbol{\Gamma}_{1}^{2}+\operatorname{tr} \boldsymbol{\Gamma}_{2}^{2}-2 \operatorname{tr} \boldsymbol{\Gamma}_{1} \boldsymbol{\Gamma}_{2}
$$

Again, high dimensionality is a serious problem because the sample spectral moments, $\operatorname{tr} \widehat{\boldsymbol{\Gamma}}_{i}^{j} ; i=1,2$, are poor estimates of the corresponding moments $\operatorname{tr} \boldsymbol{\Gamma}_{i}^{j} ; i=1,2$.

Schott (2007) obtains the so called $t_{T n}$ statistic which is an unbiased measure of the distance in (12). Assuming the data to be i.i.d. normal, the sample covariance matrix conforms to a Wishart distribution, $T \hat{\boldsymbol{\Gamma}}_{i} \sim$ $W_{n}\left(\boldsymbol{\Gamma}_{i} ; T\right)$, and the $t_{T n}$ statistic (defined in expression 1, p. 6536, of Schott, 2007 ) converges, under the null, to a normal distribution with mean 0 and a well-defined finite variance $\left(\theta^{2}\right.$ detailed in expression 4 , p. 6538). Asymptotic normality of $t_{T n}$ holds if $T_{i}$ and $n$ approach infinity at a regular rate (that is, 
$\lim _{h \rightarrow \infty} \frac{n}{T_{i h}}=b_{i}$ where $b_{i} \in[0 ; \infty)$ and $b_{i}>0$ for at least one $\left.i\right)$. Again the test can be extended to the case of comparing $d$ breakpoints. The Schott test has been refined recently by Srivastava et al. (2014) into the $T_{3}$ test, which is based on more efficient sampling estimates of spectral moments; also, it is robust to departure from normality.

Srivastava (2007) uses a lower bound of the Frobenius norm: $\operatorname{tr}\left(\boldsymbol{\Gamma}_{1}-\boldsymbol{\Gamma}_{2}\right)^{2} \geqslant$ $\left(\sqrt{\operatorname{tr} \Gamma_{1}^{2}}-\sqrt{\operatorname{tr} \Gamma_{2}^{2}}\right)^{2} \geqslant 0$, testing that $\operatorname{tr} \Gamma_{1}^{2}-\operatorname{tr} \boldsymbol{\Gamma}_{2}^{2}=0$. Like Schott (2007), he considers consistent estimates of the traces of both matrices and a consistent estimate of the variance of the difference of the traces (more details in Srivastava, 2007). The statistic obtained, called $T_{2}$, is asymptotically distributed $\mathrm{N}(0,1)$ under the same conditions as in Schott's $t_{T n}$, that is $(T ; n) \rightarrow \infty$, and i.i.d. normality. In Srivastava and Yanagihara (2010) the $T_{2}$ statistic is transformed into a standardized version, called $Q_{2}$, defined as $\operatorname{tr} \boldsymbol{\Gamma}_{1}^{2} /\left(\operatorname{tr} \boldsymbol{\Gamma}_{1}\right)^{2}-\operatorname{tr} \boldsymbol{\Gamma}_{2}^{2} /\left(\operatorname{tr} \boldsymbol{\Gamma}_{2}\right)^{2}$. Both test statistics, $Q_{2}$ and $T_{2}$, can also be extended to the $d$ breakpoints case.

Li and Chen (2012) address the 'large n, small T' question using a different, more direct approach. They propose to streamline terms in the traces, $\operatorname{tr} \widehat{\boldsymbol{\Gamma}}_{i}^{j} ; i=1,2$, so as to make unbiased estimators by using $U$ statistics. In fact, the statistic that they propose is an estimate of the trace of (12), $L_{T_{1} ; T_{2}}=A_{T_{1}}+A_{T_{2}}-2 C_{T_{1} ; T_{2}}$ where $A_{T i}$ and $C_{T_{1} ; T_{2}}$ are unbiased estimates of the respective traces (details in Li and Chen, 2012, p. 910-911). Under the null, $L_{T_{1} ; T_{2}}$ is centred on zero, has a well-defined finite variance, $\sigma_{T_{1} ; T_{2}}^{2}$, and supports a CLT so that $L_{T_{1} ; T_{2}}=\frac{T_{T_{1} ; T_{2}}}{\sigma_{T_{1} ; T_{2}}} \rightarrow \stackrel{D}{D}(0,1)$. Note that the $L_{T_{1} ; T_{2}}$ of $\mathrm{Li}$ and Chen (2012) does not require the assumption of normality but is restricted to the case of a single breakpoint.

Finally, the approach of García (2012) is based on the eigenvectors of the two covariance matrices. The idea is that, under the null, the eigenvectors obtained for either of the two sample covariance matrices will explain a similar amount of variation in either of the two samples. Denote by $\mathbf{E}_{1}$ and $\mathbf{E}_{2}$ the orthonormal matrices of eigenvectors corresponding to $\hat{\Gamma}_{1}$ and $\hat{\boldsymbol{\Gamma}}_{2}$, which may be combined with the matrices of data observed in both subsamples, $\mathbf{y}_{j}$ (or the residuals, $\hat{\mathbf{u}}_{j}$, if a SEM model has been previously estimated) to form the four matrices:

$$
\mathbf{P}_{j k}=\left[\mathbf{p}_{1 j k}, \mathbf{p}_{2 j k}, \ldots, \mathbf{p}_{n j k}\right]=\mathbf{y}_{j} \mathbf{E}_{k} ; \quad j, k=1,2
$$

The eigenvalues of $\hat{\boldsymbol{\Gamma}}_{1}$ and $\hat{\boldsymbol{\Gamma}}_{2}$ are calculated as the sums of squares of 
elements of $\mathbf{p}_{j j},(j=1,2)$. Now define the sums of squares of the elements of the columns of $\mathbf{P}_{j k}$ to be $\left\{v_{i j k}, i=1,2, \ldots, n\right\}$ and a new measure of distance between the two covariance matrices:

$$
S_{1}=2 \sum_{i=1}^{n}\left[\left(v_{i 11}-v_{i 21}\right)^{2}+\left(v_{i 12}-v_{i 22}\right)^{2}\right]
$$

No distribution has been provided for $S_{1}$. Garcia (2012) proposes the following resampling permutation approach:

(i) Obtain the statistic $S_{1}$ for the observed data.

(ii) Resample with replacement, under the null of (9); take $50 \%$ of the resampled observations from the first sub-sample, the other $50 \%$ from the second sub-sample, and reassign them randomly to the first or to the second sub-sample.

(iii) Calculate the sequence of resampled statistics $\left\{S_{1}^{g}, g=1,2, \ldots, G\right\}$, $G$ being the number of resampled samples.

(iv) Compute the empirical p-value of the resampling experiment as:

$$
\operatorname{pval}\left(S_{1}\right)=\frac{1}{G} \sum_{g=1}^{G} \mathbb{I}\left(S_{1}^{g}>S_{1}\right)
$$

where $\mathbb{I}(A)$ is the indicator function of event, $A$.

(v) Reject the null hypothesis of equal covariance matrices, (9), if: $\operatorname{pval}\left(S_{1}\right)<$ $\alpha$ for some chosen nominal significance level, $\alpha$.

In case of rejecting the null, we may use the statistics $S_{2}$ and $S_{3}$ (defined in Garcia, 2012) to assess the characteristics of the break. $S_{3}$ evaluates the proportion of the break due to heteroskedasticity whereas $S_{2}$ measures the proportion attributable to changes in the covariances among the individuals.

\section{Monte Carlo evidence}

Our experimental DGP is a spatial panel data model, whose weights matrix remains stable under the null; it may be either a spatial error model, SEM, or a spatial lag model, SLM. In the first case:

$$
\mathbf{y}_{t}=\boldsymbol{\mu}_{t}+\mathbf{x}_{t} \boldsymbol{\beta}+\left(\mathbf{I}-\rho_{u} \mathbf{W}\right)^{-1} \mathbf{u}_{t} ; \quad t=1,2,3, \ldots T
$$

The model of the alternative hypothesis incorporates two SEM equations with different weighting matrices (similarly for the SLM case): 


$$
\begin{gathered}
\mathbf{y}_{t}=\boldsymbol{\mu}_{t}+\mathbf{x}_{t} \boldsymbol{\beta}+\left(\mathbf{I}-\rho_{u} \mathbf{W}_{1}\right)^{-1} \mathbf{u}_{t} ; \quad t=1,2, \ldots T_{b} \\
\mathbf{y}_{t}=\boldsymbol{\mu}_{t}+\mathbf{x}_{t} \boldsymbol{\beta}+\left(\mathbf{I}-\rho_{u} \mathbf{W}_{2}\right)^{-1} \mathbf{u}_{t} ; \quad t=T_{b}+1, \ldots T \\
\mathbf{W}_{1} \neq \mathbf{W}_{2}
\end{gathered}
$$

The list of cases from which we extract issues for discussion comprises small to medium sample sizes in $n$ and medium to large sample sizes in $T$, allowing for cases of high dimensionality. The tests are expected to work better with increasing $T$. Bai et al. (2009) report results with very large values of $T$, up to 12,800 observations, which confirm the consistency of their $T_{N}$ test; however this will seldom be realistic in applied work. Schott (2007), on the contrary, simulates very short time spans, $T=4$, with serious dimensionality contraints $(n=128)$. The parameters for our Monte Carlo are the following:

- Time-dimension: sample sizes $T=\{10 ; 30 ; 60 ; 150 ; 250 ; 500\}$.

- Space-dimension: sample sizes $n=\{16 ; 36 ; 64 ; 144 ; 225 ; 400\}$.

- Regional shape: regular square lattice with side $n^{1 / 2}$.

- Weighting matrices. The weights matrix for the first period follows a rook contiguity pattern, $\mathbf{W}^{R}$. We distinguish two cases for the second period under the alternative: a queen contiguity pattern, $\mathbf{W}^{Q}$, and a pattern based on the inverse of the distances between the centroids of the cells, $\mathbf{W}^{D}$. The three matrices are row-standardized. The rook pattern is more akin to the queen case (this is a soft change in the weights in terms of Lee and $\mathrm{Yu}, 2012$ ) than to the inverse of the distance; i.e., the Frobenius norm of the matrix $\mathbf{W}^{R}-\mathbf{W}^{Q}, n=16$, is 1.50 but 1.75 in the case of $\mathbf{W}^{R}-\mathbf{W}^{D}$.

- Known or unknown breakpoints. The breakpoints, $T_{b}$, are located in the middle of the time sequence. Then we simulate two cases. In the first, it is supposed that we know where the breakpoint is located whereas this point is unknown in the second. In that case, the tests are obtained following a rolling process centred on $T_{b}$; the sequence includes $20 \%$ of the central observations according to the examples in Table $1\left(T_{a}\right.$ and $T_{c}$ are the starting and ending points for the rolling sequence): 


\begin{tabular}{|c|c|c|c|}
\hline \multicolumn{4}{|c|}{ Table 1: Sample size, location of breakpoint,and testing intervals } \\
\hline$T$ & $T_{a}$ & $T_{b}$ & $T_{c}$ \\
\hline $\mathbf{6 0}$ & 24 & 30 & 36 \\
\hline $\mathbf{1 5 0}$ & 61 & 75 & 90 \\
\hline $\mathbf{5 0 0}$ & 201 & 250 & 300 \\
\hline
\end{tabular}

- Nature of the break, instantaneous or gradual. In the first case, the break takes place as in (17). For the second case, we define a 'breaking period' as the time span along which change is happening; this period is equal to $10 \%$ of the sample, $T^{b p}=0.1 \times T$. The weighting matrix that intervenes in the DGP for this transitional interval changes gradually in each period according to $\mathbf{W}^{b p}(t)=\frac{T_{b}-t}{T^{b p}} \times \mathbf{W}^{R}+\left(1-\frac{T_{b}-t}{T^{b p}}\right) \times \mathbf{W}^{i}$ with $i \in\{Q, D\}$. That is, the transition from one matrix to the other begins in period $\left(T_{b}-T^{b p}\right)$ and it is not fully completed until $T_{b}$.

- Knowledge about the DGP. Generally, the DGP remains unkown to the user so the tests have been obtained for the covariance matrix of y. Assuming that the spatial structure appears only in the errors of the equation (that is, is a SEM model) and that this is known, the user can benefit from a previous consistent estimation of the mean equation of the model, from which the corresponding (consistent) residuals can be obtained. In this framework, the tests should be applied to the covariance matrix of the residuals. Results are briefly discussed in Appendix A.

- Spatial parameter values $\rho \in\{0.1 ; 0.3 ; 0.5 ; 0.7 ; 0.9\}$

- Unobserved individual and idiosyncratic disturbances are drawn from standard normal distributions: $\mu_{i} \sim i . i . d . N\left(0, \sigma_{\mu}^{2}\right), u_{i t} \sim i . i . d . N\left(0, \sigma_{u}^{2}\right)$, $\sigma_{\mu}^{2}=1, \sigma_{u}^{2}=1$

- Exogenous regressor dimension, $k=3 ; x_{t i 1}=1 ; x_{t i 2}$ and $x_{t i 3}$ drawn from $N\left(0 ; I_{2}\right)$ for $t=1,2, \ldots, T ; i=1,2, \ldots, n$.

- Regression parameter values $\boldsymbol{\beta}^{\prime}=\left(1,1, \beta_{3}\right)$. The value of $\beta_{3}$ has been defined in order to assure a given signal-to-noise ratio in the pooled regression (with no spatial effects) according to the following Table: 


\begin{tabular}{|c|c|c|c|}
\hline \multicolumn{4}{|c|}{ Table 2: Expected $R^{2}$ and value of $\beta_{3}$} \\
\hline & $R^{2} \simeq 0.5$ & $R^{2} \simeq 0.6$ & $R^{2} \simeq 0.8$ \\
\hline$\beta_{3}=\sqrt{\frac{\beta_{2}^{2}\left(1-R^{2}\right) \sigma_{x_{2}}^{2}+R^{2}\left(\sigma_{u}^{2}+\sigma_{\mu}^{2}\right)}{\left(1-R^{2}\right) \sigma_{x_{3}}^{2}}}$ & 1.7 & 2 & 3 \\
\hline
\end{tabular}

- For estimating size and power, we use 1,000 replications, which produce approximate standard errors for the $10 \%, 5 \%$ and $1 \%$ significance level of $0.00948,0.00690$ and 0.00314 respectively.

- Two of the tests, the $L R$ of (10) and the $T_{N}$ of (11) are based on the likelihood ratio approach, five other tests (Schott's $t_{T_{n}}$, the $T_{3}$ refined version of Srivastava et al., Srivastava's $T_{2}$, Srivastava and Yanagihara's $Q_{2}$ and $\mathrm{Li}$ and Chen's $L_{T_{1} ; T_{2}}$ ) are based on the squared Frobenius norm and, finally, there is Garcia's $S_{1}$ bootstrap test. For the likelihood ratio tests we need a concentration index smaller than $1,\left(c_{i}=\frac{n}{T_{i}}<1\right)$ so there are cases where the LR based tests cannot be obtained (the same applies for Garcia's $S_{1}$ ); this is not the case for the Frobenius norm based tests.

\subsection{Results of the Monte Carlo}

Tables 3 and 4 and Figures 1 to 3 summarize the main results of the Monte $\mathrm{Carlo}^{2}$. The Tables correspond to the case of a breakpoint with known location whereas the Figures describe the case of a breakpoint with unknown location. The data that appear in the Tables are the percentage of rejections of the null hypothesis of (9), using the corresponding test. Figures represent the evolution of the test values over the testing interval in the two cases of instantaneous and progressive break in the weights matrix.

Tables 3a,b report estimated size, whereas Tables 4a,b,c,d show estimated power. The impact of the signal-to-noise-ratio is really small, both in size and power. Results tend to be slightly better for the case of a high signalto-noise ratio but the difference is always below 0.05 points, so we focus on the case of a high signal-to-noise ratio.

\footnotetext{
${ }^{2}$ For the sake of brevity, we omit the details for the cases not shown in the Tables. The main tendencies, both in size as well in power, are maintained. Full details are available from the authors, upon request
} 


\begin{tabular}{|c|c|c|c|c|c|c|c|c|c|c|c|c|}
\hline \multicolumn{13}{|c|}{$\begin{array}{c}\text { Table 3a: Estimated size (significance level=0.05). Known breakpoint. SEM process } \\
\text { High signal-to-noise ratio. Matrix in the DGP: WR. }\end{array}$} \\
\hline \multicolumn{13}{|c|}{$\rho=0.3$} \\
\hline$T$ & \multicolumn{3}{|c|}{30} & \multicolumn{3}{|c|}{60} & \multicolumn{3}{|c|}{150} & \multicolumn{3}{|c|}{250} \\
\hline $\mathrm{n}$ & 16 & 144 & 225 & 16 & 144 & 225 & 16 & $144^{*}$ & 225 & 16 & $144^{*}$ & 225 \\
\hline LR & - & - & - & 0.84 & - & - & 0.62 & 0.75 & - & 0.34 & 0.41 & - \\
\hline $\mathbf{T}_{\mathbf{N}}$ & - & - & - & 0.14 & - & - & 0.07 & 0.20 & - & 0.06 & 0.10 & - \\
\hline $\mathbf{t}_{\mathbf{T n}}$ & 0.03 & 0.03 & 0.04 & 0.05 & 0.03 & 0.02 & 0.06 & 0.04 & 0.06 & 0.06 & 0.04 & 0.04 \\
\hline $\mathbf{L}_{\mathrm{T} 1, \mathrm{~T} 2}$ & 0.27 & 0.45 & 0.61 & 0.19 & 0.29 & 0.35 & 0.16 & 0.25 & 0.30 & 0.14 & 0.22 & 0.26 \\
\hline $\mathbf{T}_{2}$ & 0.00 & 0.00 & 0.03 & 0.01 & 0.00 & 0.00 & 0.02 & 0.00 & 0.00 & 0.02 & 0.00 & 0.00 \\
\hline $\mathbf{T}_{3}$ & 0.01 & 0.02 & 0.09 & 0.05 & 0.00 & 0.00 & 0.06 & 0.00 & 0.00 & 0.06 & 0.02 & 0.01 \\
\hline $\mathbf{Q}_{2}$ & 0.00 & 0.00 & 0.02 & 0.00 & 0.00 & 0.00 & 0.00 & 0.00 & 0.00 & 0.00 & 0.00 & 0.00 \\
\hline $\mathbf{S}_{1}$ & - & - & - & 0.62 & - & - & 0.46 & 0.61 & - & 0.41 & 0.55 & - \\
\hline \multicolumn{13}{|c|}{$\rho=0.5$} \\
\hline $\mathbf{T}$ & \multicolumn{3}{|c|}{30} & \multicolumn{3}{|c|}{60} & \multicolumn{3}{|c|}{150} & \multicolumn{3}{|c|}{250} \\
\hline $\mathbf{n}$ & 16 & 144 & 225 & 16 & 144 & 225 & 16 & $144^{*}$ & 225 & 16 & $144^{*}$ & 225 \\
\hline LR & - & - & - & 0.86 & - & - & 0.59 & 0.71 & - & 0.31 & 0.38 & - \\
\hline $\mathbf{T}_{\mathbf{N}}$ & - & - & - & 0.13 & - & - & 0.07 & 0.21 & - & 0.05 & 0.10 & - \\
\hline $\mathbf{t}_{\mathbf{T n}}$ & 0.04 & 0.03 & 0.03 & 0.05 & 0.05 & 0.04 & 0.07 & 0.04 & 0.04 & 0.06 & 0.06 & 0.04 \\
\hline $\mathbf{L}_{\mathrm{T} 1, \mathrm{~T} 2}$ & 0.22 & 0.41 & 0.59 & 0.21 & 0.25 & 0.38 & 0.17 & 0.27 & 0.27 & 0.11 & 0.19 & 0.24 \\
\hline $\mathbf{T}_{2}$ & 0.01 & 0.00 & 0.03 & 0.01 & 0.00 & 0.00 & 0.02 & 0.01 & 0.00 & 0.04 & 0.00 & 0.01 \\
\hline $\mathbf{T}_{\mathbf{3}}$ & 0.02 & 0.01 & 0.08 & 0.04 & 0.00 & 0.00 & 0.05 & 0.00 & 0.00 & 0.06 & 0.01 & 0.01 \\
\hline $\mathbf{Q}_{2}$ & 0.00 & 0.01 & 0.01 & 0.00 & 0.00 & 0.00 & 0.00 & 0.00 & 0.00 & 0.00 & 0.00 & 0.00 \\
\hline $\mathbf{S}_{1}$ & - & - & - & 0.56 & - & - & 0.49 & 0.58 & - & 0.44 & 0.51 & - \\
\hline \multicolumn{13}{|c|}{$\rho=0.7$} \\
\hline $\mathbf{T}$ & \multicolumn{3}{|c|}{30} & \multicolumn{3}{|c|}{60} & \multicolumn{3}{|c|}{150} & \multicolumn{3}{|c|}{250} \\
\hline $\mathrm{n}$ & 16 & 144 & 225 & 16 & 144 & 225 & 16 & $144^{*}$ & 225 & 16 & $144^{*}$ & 225 \\
\hline LR & - & - & - & 0.81 & - & - & 0.42 & 0.55 & - & 0.18 & 0.22 & - \\
\hline $\mathbf{T}_{\mathbf{N}}$ & - & - & - & 0.10 & - & - & 0.07 & 0.22 & - & 0.06 & 0.09 & - \\
\hline $\mathbf{t}_{\mathbf{T n}}$ & 0.04 & 0.04 & 0.02 & 0.04 & 0.04 & 0.04 & 0.06 & 0.05 & 0.05 & 0.07 & 0.05 & 0.05 \\
\hline $\mathbf{L}_{\mathrm{T} 1, \mathrm{~T} 2}$ & 0.20 & 0.42 & 0.52 & 0.20 & 0.20 & 0.32 & 0.15 & 0.22 & 0.20 & 0.13 & 0.18 & 0.23 \\
\hline $\mathbf{T}_{2}$ & 0.01 & 0.01 & 0.01 & 0.02 & 0.00 & 0.00 & 0.02 & 0.00 & 0.00 & 0.04 & 0.01 & 0.00 \\
\hline $\mathbf{T}_{\mathbf{3}}$ & 0.01 & 0.00 & 0.08 & 0.04 & 0.00 & 0.00 & 0.06 & 0.00 & 0.00 & 0.07 & 0.02 & 0.01 \\
\hline $\mathbf{Q}_{2}$ & 0.00 & 0.01 & 0.01 & 0.02 & 0.01 & 0.00 & 0.01 & 0.00 & 0.01 & 0.02 & 0.00 & 0.00 \\
\hline $\mathbf{S}_{1}$ & - & - & - & 0.44 & - & - & 0.42 & 0.55 & - & 0.39 & 0.53 & - \\
\hline
\end{tabular}




\begin{tabular}{|c|c|c|c|c|c|c|c|c|c|c|c|c|}
\hline \multicolumn{13}{|c|}{$\begin{array}{l}\text { Table 3b: Estimated size (significance level=0.05). Known breakpoint. SLM process } \\
\text { High signal-to-noise ratio. Matrix in the DGP: WR. }\end{array}$} \\
\hline \multicolumn{13}{|c|}{$\rho=0.3$} \\
\hline $\mathbf{T}$ & \multicolumn{3}{|c|}{30} & \multicolumn{3}{|c|}{60} & \multicolumn{3}{|c|}{150} & \multicolumn{3}{|c|}{250} \\
\hline $\mathbf{n}$ & 16 & 144 & 225 & 16 & 144 & 225 & 16 & $144^{*}$ & 225 & 16 & $144 *$ & 225 \\
\hline LR & - & - & - & 0.88 & - & - & 0.66 & 0.76 & - & 0.41 & 0.48 & - \\
\hline $\mathbf{T}_{\mathbf{N}}$ & - & - & - & 0.11 & - & - & 0.08 & 0.21 & - & 0.05 & 0.07 & - \\
\hline $\mathbf{t}_{\mathrm{Tn}}$ & 0.05 & 0.02 & 0.03 & 0.06 & 0.04 & 0.03 & 0.07 & 0.05 & 0.05 & 0.06 & 0.05 & 0.05 \\
\hline $\mathbf{L}_{\mathrm{T1}, \mathrm{T} 2}$ & 0.35 & 0.52 & 0.73 & 0.29 & 0.32 & 0.38 & 0.24 & 0.29 & 0.32 & 0.19 & 0.20 & 0.28 \\
\hline $\mathbf{T}_{2}$ & 0.01 & 0.00 & 0.02 & 0.02 & 0.00 & 0.00 & 0.03 & 0.04 & 0.03 & 0.05 & 0.04 & 0.03 \\
\hline $\mathbf{T}_{3}$ & 0.03 & 0.00 & 0.10 & 0.05 & 0.00 & 0.00 & 0.06 & 0.01 & 0.00 & 0.05 & 0.02 & 0.01 \\
\hline $\mathbf{Q}_{2}$ & 0.00 & 0.00 & 0.00 & 0.00 & 0.00 & 0.00 & 0.01 & 0.00 & 0.00 & 0.01 & 0.00 & 0.00 \\
\hline $\mathbf{S}_{1}$ & - & - & - & 0.66 & - & - & 0.48 & 0.65 & - & 0.43 & 0.54 & - \\
\hline \multicolumn{13}{|c|}{$\rho=0.5$} \\
\hline $\mathbf{T}$ & \multicolumn{3}{|c|}{30} & \multicolumn{3}{|c|}{60} & \multicolumn{3}{|c|}{150} & \multicolumn{3}{|c|}{250} \\
\hline $\mathbf{n}$ & 16 & 144 & 225 & 16 & 144 & 225 & 16 & $144^{*}$ & 225 & 16 & $144 *$ & 225 \\
\hline LR & - & - & - & 0.82 & - & - & 0.65 & 0.71 & - & 0.51 & 0.60 & - \\
\hline $\mathbf{T}_{\mathbf{N}}$ & - & - & - & 0.12 & - & - & 0.06 & 0.23 & - & 0.05 & 0.09 & - \\
\hline $\mathbf{t}_{\mathrm{Tn}}$ & 0.05 & 0.03 & 0.04 & 0.06 & 0.04 & 0.04 & 0.07 & 0.05 & 0.05 & 0.07 & 0.05 & 0.05 \\
\hline $\mathbf{L}_{\mathrm{T} 1, \mathrm{~T} 2}$ & 0.35 & 0.39 & 0.51 & 0.28 & 0.32 & 0.35 & 0.31 & 0.35 & 0.39 & 0.19 & 0.28 & 0.36 \\
\hline $\mathbf{T}_{2}$ & 0.02 & 0.00 & 0.00 & 0.02 & 0.00 & 0.00 & 0.05 & 0.00 & 0.00 & 0.05 & 0.01 & 0.01 \\
\hline $\mathbf{T}_{\mathbf{3}}$ & 0.04 & 0.00 & 0.13 & 0.05 & 0.00 & 0.00 & 0.07 & 0.02 & 0.00 & 0.07 & 0.02 & 0.02 \\
\hline $\mathbf{Q}_{2}$ & 0.00 & 0.00 & 0.00 & 0.00 & 0.00 & 0.00 & 0.02 & 0.00 & 0.00 & 0.02 & 0.00 & 0.00 \\
\hline $\mathbf{S}_{1}$ & - & - & - & 0.59 & - & - & 0.39 & 0.47 & - & 0.40 & 0.48 & - \\
\hline \multicolumn{13}{|c|}{$\rho=0.7$} \\
\hline $\mathbf{T}$ & \multicolumn{3}{|c|}{30} & \multicolumn{3}{|c|}{60} & \multicolumn{3}{|c|}{150} & \multicolumn{3}{|c|}{250} \\
\hline $\mathbf{n}$ & 16 & 144 & 225 & 16 & 144 & 225 & 16 & $144^{*}$ & 225 & 16 & $144 *$ & 225 \\
\hline LR & - & - & - & 0.79 & - & - & 0.36 & 0.46 & - & 0.27 & 0.31 & - \\
\hline $\mathbf{T}_{\mathbf{N}}$ & - & - & - & 0.13 & - & - & 0.07 & 0.24 & - & 0.05 & 0.08 & - \\
\hline $\mathbf{t}_{\mathrm{Tn}}$ & 0.06 & 0.04 & 0.03 & 0.07 & 0.04 & 0.04 & 0.08 & 0.05 & 0.04 & 0.08 & 0.05 & 0.05 \\
\hline $\mathbf{L}_{\mathrm{T} 1, \mathrm{~T} 2}$ & 0.28 & 0.31 & 0.48 & 0.29 & 0.37 & 0.39 & 0.24 & 0.29 & 0.33 & 0.19 & 0.23 & 0.27 \\
\hline $\mathbf{T}_{2}$ & 0.02 & 0.01 & 0.00 & 0.03 & 0.01 & 0.00 & 0.05 & 0.01 & 0.01 & 0.03 & 0.03 & 0.02 \\
\hline $\mathbf{T}_{3}$ & 0.08 & 0.00 & 0.00 & 0.07 & 0.01 & 0.00 & 0.09 & 0.03 & 0.01 & 0.09 & 0.04 & 0.03 \\
\hline $\mathbf{Q}_{2}$ & 0.00 & 0.00 & 0.00 & 0.02 & 0.00 & 0.00 & 0.02 & 0.02 & 0.01 & 0.02 & 0.01 & 0.02 \\
\hline $\mathbf{S}_{1}$ & - & - & - & 0.46 & - & - & 0.27 & 0.31 & - & 0.20 & 0.24 & - \\
\hline
\end{tabular}




\begin{tabular}{|c|c|c|c|c|c|c|c|c|c|c|c|c|}
\hline \multicolumn{13}{|c|}{$\begin{array}{l}\text { Table 4a: Estimated power (significance level=0.05). Known breakpoint. SEM process } \\
\text { High signal-to-noise ratio. Matrices in the DGP: WR vs WD }\end{array}$} \\
\hline \multicolumn{13}{|c|}{$\rho=0.3$} \\
\hline $\mathbf{T}$ & \multicolumn{3}{|c|}{30} & \multicolumn{3}{|c|}{60} & \multicolumn{3}{|c|}{150} & \multicolumn{3}{|c|}{250} \\
\hline $\mathrm{n}$ & 16 & 144 & 225 & 16 & 144 & 225 & 16 & $144 *$ & 225 & 16 & $144^{*}$ & 225 \\
\hline $\mathbf{T}_{\mathrm{N}}$ & - & - & - & 0.16 & - & - & 0.37 & 0.51 & - & 0.51 & 0.72 & - \\
\hline $\mathbf{t}_{\mathbf{T n}}$ & 0.13 & 0.23 & 0.34 & 0.14 & 0.44 & 0.54 & 0.47 & 0.61 & 0.68 & 0.59 & 0.70 & 0.75 \\
\hline $\mathbf{T}_{2}$ & 0.08 & 0.09 & 0.21 & 0.10 & 0.15 & 0.26 & 0.18 & 0.27 & 0.30 & 0.25 & 0.31 & 0.41 \\
\hline \multicolumn{13}{|c|}{$\rho=0.5$} \\
\hline $\mathbf{T}$ & \multicolumn{3}{|c|}{30} & \multicolumn{3}{|c|}{60} & \multicolumn{3}{|c|}{150} & \multicolumn{3}{|c|}{250} \\
\hline $\mathrm{n}$ & 16 & 144 & 225 & 16 & 144 & 225 & 16 & $144^{*}$ & 225 & 16 & $144^{*}$ & 225 \\
\hline $\mathbf{T}_{\mathbf{N}}$ & - & - & - & 0.35 & - & - & 0.46 & 0.63 & - & 0.60 & 0.78 & - \\
\hline $\mathbf{t}_{\mathbf{T n}}$ & 0.15 & 0.31 & 0.52 & 0.25 & 0.49 & 0.60 & 0.51 & 0.58 & 0.71 & 0.67 & 0.75 & 0.86 \\
\hline $\mathbf{T}_{2}$ & 0.10 & 0.10 & 0.27 & 0.12 & 0.19 & 0.40 & 0.25 & 0.31 & 0.51 & 0.34 & 0.53 & 0.62 \\
\hline \multicolumn{13}{|c|}{$\rho=0.7$} \\
\hline $\mathbf{T}$ & \multicolumn{3}{|c|}{30} & \multicolumn{3}{|c|}{60} & \multicolumn{3}{|c|}{150} & \multicolumn{3}{|c|}{250} \\
\hline $\mathrm{n}$ & 16 & 144 & 225 & 16 & 144 & 225 & 16 & $144 *$ & 225 & 16 & $144^{*}$ & 225 \\
\hline $\mathbf{T}_{\mathbf{N}}$ & - & - & - & 0.48 & - & - & 0.62 & 0.73 & - & 0.79 & 0.88 & - \\
\hline $\mathbf{t}_{\mathbf{T n}}$ & 0.18 & 0.43 & 0.63 & 0.27 & 0.54 & 0.65 & 0.47 & 0.66 & 0.75 & 0.43 & 0.76 & 0.92 \\
\hline $\mathbf{T}_{2}$ & 0.10 & 0.16 & 0.33 & 0.15 & 0.22 & 0.45 & 0.19 & 0.44 & 0.62 & 0.37 & 0.71 & 0.81 \\
\hline
\end{tabular}

\begin{tabular}{|c|c|c|c|c|c|c|c|c|c|c|c|c|}
\hline \multicolumn{13}{|c|}{$\begin{array}{c}\text { Table 4b: Estimated power (significance level=0.05). Known breakpoint. SLM process } \\
\text { High signal-to-noise ratio. Matrices in the DGP: WR vs WD. }\end{array}$} \\
\hline \multicolumn{13}{|c|}{$\rho=0.3$} \\
\hline $\mathbf{T}$ & \multicolumn{3}{|c|}{30} & \multicolumn{3}{|c|}{60} & \multicolumn{3}{|c|}{150} & \multicolumn{3}{|c|}{250} \\
\hline $\mathrm{n}$ & 16 & 144 & 225 & 16 & 144 & 225 & 16 & $144 *$ & 225 & 16 & $144 *$ & 225 \\
\hline $\mathbf{T}_{\mathrm{N}}$ & - & - & - & 0.17 & - & - & 0.26 & 0.46 & - & 0.54 & 0.85 & - \\
\hline $\mathbf{t}_{\mathbf{T n}}$ & 0.07 & 0.16 & 0.26 & 0.11 & 0.27 & 0.44 & 0.31 & 0.56 & 0.58 & 0.62 & 0.92 & 0.92 \\
\hline $\mathbf{T}_{2}$ & 0.04 & 0.14 & 0.31 & 0.10 & 0.27 & 0.48 & 0.31 & 0.96 & 0.98 & 0.50 & 1.00 & 1.00 \\
\hline \multicolumn{13}{|c|}{$\rho=0.5$} \\
\hline $\mathbf{T}$ & \multicolumn{3}{|c|}{30} & \multicolumn{3}{|c|}{60} & \multicolumn{3}{|c|}{150} & \multicolumn{3}{|c|}{250} \\
\hline $\mathrm{n}$ & 16 & 144 & 225 & 16 & 144 & 225 & 16 & $144^{*}$ & 225 & 16 & $144^{*}$ & 225 \\
\hline $\mathbf{T}_{\mathrm{N}}$ & - & - & - & 0.35 & - & - & 0.83 & 0.87 & - & 0.99 & 1.00 & - \\
\hline$t_{T n}$ & 0.17 & 0.33 & 0.53 & 0.32 & 0.83 & 0.87 & 0.83 & 1.00 & 1.00 & 0.99 & 1.00 & 1.00 \\
\hline $\mathbf{T}_{2}$ & 0.11 & 0.46 & 0.58 & 0.26 & 1.00 & 1.00 & 0.53 & 1.00 & 1.00 & 0.74 & 1.00 & 1.00 \\
\hline \multicolumn{13}{|c|}{$\rho=0.7$} \\
\hline $\mathbf{T}$ & \multicolumn{3}{|c|}{30} & \multicolumn{3}{|c|}{60} & \multicolumn{3}{|c|}{150} & \multicolumn{3}{|c|}{250} \\
\hline $\mathrm{n}$ & 16 & 144 & 225 & 16 & 144 & 225 & 16 & 144* & 225 & 16 & 144* & 225 \\
\hline $\mathbf{T}_{\mathbf{N}}$ & - & - & - & 0.75 & - & - & 1.00 & 1.00 & - & 1.00 & 1.00 & - \\
\hline $\mathbf{t}_{T \mathbf{n}}$ & 0.19 & 0.93 & 0.98 & 0.34 & 1.00 & 1.00 & 0.90 & 1.00 & 1.00 & 1.00 & 1.00 & 1.00 \\
\hline $\mathbf{T}_{2}$ & 0.10 & 0.86 & 0.95 & 0.15 & 1.00 & 1.00 & 0.27 & 1.00 & 1.00 & 0.35 & 1.00 & 1.00 \\
\hline
\end{tabular}




\begin{tabular}{|c|c|c|c|c|c|c|c|c|c|c|c|c|}
\hline \multicolumn{13}{|c|}{$\begin{array}{c}\text { Table 4c: Estimated power (significance level=0.05). Known breakpoint. SEM process } \\
\text { High signal-to-noise ratio. Matrices in the DGP: WR vs WQ }\end{array}$} \\
\hline \multicolumn{13}{|c|}{$\rho=0.3$} \\
\hline $\mathbf{T}$ & \multicolumn{3}{|c|}{30} & \multicolumn{3}{|c|}{60} & \multicolumn{3}{|c|}{150} & \multicolumn{3}{|c|}{250} \\
\hline $\mathbf{n}$ & 16 & 144 & 225 & 16 & 144 & 225 & 16 & $144 *$ & 225 & 16 & $144 *$ & 225 \\
\hline $\mathbf{T}_{\mathbf{N}}$ & - & - & - & 0.16 & - & - & 0.37 & 0.51 & - & 0.51 & 0.72 & - \\
\hline $\mathbf{t}_{\mathbf{T n}}$ & 0.08 & 0.14 & 0.27 & 0.17 & 0.25 & 0.31 & 0.24 & 0.32 & 0.39 & 0.27 & 0.42 & 0.58 \\
\hline $\mathbf{T}_{2}$ & 0.09 & 0.10 & 0.16 & 0.12 & 0.16 & 0.24 & 0.15 & 0.21 & 0.25 & 0.19 & 0.25 & 0.33 \\
\hline \multicolumn{13}{|c|}{$\rho=0.5$} \\
\hline $\mathbf{T}$ & \multicolumn{3}{|c|}{30} & \multicolumn{3}{|c|}{60} & \multicolumn{3}{|c|}{150} & \multicolumn{3}{|c|}{250} \\
\hline $\mathbf{n}$ & 16 & 144 & 225 & 16 & 144 & 225 & 16 & $144^{*}$ & 225 & 16 & $144 *$ & 225 \\
\hline $\mathbf{T}_{\mathbf{N}}$ & - & - & - & 0.35 & - & - & 0.46 & 0.63 & - & 0.60 & 0.78 & - \\
\hline $\mathbf{t}_{\mathrm{Tn}}$ & 0.15 & 0.22 & 0.28 & 0.20 & 0.34 & 0.40 & 0.29 & 0.41 & 0.51 & 0.41 & 0.61 & 0.66 \\
\hline $\mathbf{T}_{2}$ & 0.10 & 0.10 & 0.17 & 0.11 & 0.14 & 0.34 & 0.16 & 0.23 & 0.37 & 0.28 & 0.35 & 0.49 \\
\hline \multicolumn{13}{|c|}{$\rho=0.7$} \\
\hline $\mathbf{T}$ & \multicolumn{3}{|c|}{30} & \multicolumn{3}{|c|}{60} & \multicolumn{3}{|c|}{150} & \multicolumn{3}{|c|}{250} \\
\hline n & 16 & 144 & 225 & 16 & 144 & 225 & 16 & $144 *$ & 225 & 16 & $144 *$ & 225 \\
\hline $\mathbf{T}_{\mathrm{N}}$ & - & - & - & 0.48 & - & - & 0.62 & 0.73 & - & 0.79 & 0.88 & - \\
\hline $\mathbf{t}_{\mathrm{Tn}}$ & 0.18 & 0.34 & 0.43 & 0.24 & 0.44 & 0.55 & 0.32 & 0.53 & 0.64 & 0.49 & 0.70 & 0.86 \\
\hline $\mathbf{T}_{2}$ & 0.10 & 0.12 & 0.30 & 0.19 & 0.23 & 0.46 & 0.19 & 0.39 & 0.56 & 0.33 & 0.61 & 0.74 \\
\hline
\end{tabular}

\begin{tabular}{|c|c|c|c|c|c|c|c|c|c|c|c|c|}
\hline \multicolumn{13}{|c|}{$\begin{array}{c}\text { Table 4d: Estimated power (significance level=0.05). Known breakpoint. SLM process } \\
\text { High signal-to-noise ratio. Matrices in the DGP: WR vs WQ }\end{array}$} \\
\hline \multicolumn{13}{|c|}{$\rho=0.3$} \\
\hline $\mathbf{T}$ & \multicolumn{3}{|c|}{30} & \multicolumn{3}{|c|}{60} & \multicolumn{3}{|c|}{150} & \multicolumn{3}{|c|}{250} \\
\hline $\mathrm{n}$ & 16 & 144 & 225 & 16 & 144 & 225 & 16 & 144* & 225 & 16 & $144^{*}$ & 225 \\
\hline $\mathbf{T}_{\mathbf{N}}$ & - & - & - & 0.17 & - & - & 0.38 & 0.53 & - & 0.47 & 0.68 & - \\
\hline $\mathbf{t}_{\mathbf{T n}}$ & 0.07 & 0.16 & 0.35 & 0.21 & 0.28 & 0.41 & 0.27 & 0.47 & 0.54 & 0.34 & 0.52 & 0.82 \\
\hline $\mathbf{T}_{2}$ & 0.08 & 0.24 & 0.31 & 0.10 & 0.37 & 0.48 & 0.32 & 0.57 & 0.67 & 0.49 & 0.67 & 0.83 \\
\hline \multicolumn{13}{|c|}{$\rho=0.5$} \\
\hline $\mathbf{T}$ & \multicolumn{3}{|c|}{30} & \multicolumn{3}{|c|}{60} & \multicolumn{3}{|c|}{150} & \multicolumn{3}{|c|}{250} \\
\hline $\mathrm{n}$ & 16 & 144 & 225 & 16 & 144 & 225 & 16 & 144* & 225 & 16 & $144 *$ & 225 \\
\hline $\mathbf{T}_{\mathbf{N}}$ & - & - & - & 0.24 & - & - & 0.46 & 0.48 & - & 0.78 & 0.74 & - \\
\hline$t_{T n}$ & 0.11 & 0.14 & 0.22 & 0.26 & 0.34 & 0.51 & 0.33 & 0.62 & 0.80 & 0.39 & 0.69 & 0.89 \\
\hline $\mathbf{T}_{2}$ & 0.12 & 0.26 & 0.35 & 0.29 & 0.40 & 0.55 & 0.46 & 0.65 & 0.84 & 0.43 & 0.82 & 0.90 \\
\hline \multicolumn{13}{|c|}{$\rho=0.7$} \\
\hline $\mathbf{T}$ & \multicolumn{3}{|c|}{30} & \multicolumn{3}{|c|}{60} & \multicolumn{3}{|c|}{150} & \multicolumn{3}{|c|}{250} \\
\hline $\mathrm{n}$ & 16 & 144 & 225 & 16 & 144 & 225 & 16 & 144* & 225 & 16 & $144 *$ & 225 \\
\hline $\mathbf{T}_{\mathbf{N}}$ & - & - & - & 0.34 & - & - & 0.74 & 0.86 & - & 0.98 & 0.99 & - \\
\hline $\mathbf{t}_{\mathbf{T n}}$ & 0.12 & 0.27 & 0.31 & 0.31 & 0.41 & 0.55 & 0.42 & 0.67 & 0.85 & 0.47 & 0.85 & 0.91 \\
\hline $\mathbf{T}_{2}$ & 0.14 & 0.26 & 0.53 & 0.15 & 0.49 & 0.61 & 0.49 & 0.70 & 0.87 & 0.65 & 0.94 & 0.96 \\
\hline
\end{tabular}


Size is a real problem for three cases: $L R, L_{T_{1} ; T_{2}}$ of $\mathrm{Li}$ and Chen and $S_{1}$ of Garcia. These tests are strongly oversized, independently of (time, spatial) sample size or the degree of spatial interaction. Data on Bai et al's $T_{N}$ test corroborate the warning of Ledoit and Wolf (2002) with respect to $L R$ type tests: these tests have a size problem when the concentration index is high. According to our results, for the $T_{N}$ test to have reasonable empirical size the ratio of time observations to spatial units should be at least 10 to 1 , so that $c_{i} \leq 0.10$. The other candidates work pretty well, especially Schott's $t_{T n}$ test whose estimated size is always around the theoretical $5 \%$ significance level. The tests using a lower bound to the Frobenius norm, $T_{2}$ and $Q_{2}$, are consistently under-sized, with size almost zero in most cases. However this is not, necessarily, a bad thing if accompanied by good power. Unfortunately, the estimated power function of the second is unacceptable (not shown in the tables) and we decided to exclude the $Q_{2}$ test from the analysis.

Another surprise comes from the $T_{3}$ test of Srivastava et al. (2014), a refinement of Schott's $t_{T n}$ test. The revision does not work in our framework where the $T_{3}$ test tends to be undersized (although for small spatial samples, its estimated size is moderately above the nominal significance level). However, like the $Q_{2}$ test, its estimated power function is unacceptable for almost all the cases (not shown in the paper), no matter sample size, spatial dependence or type of break. Consequently we have decided to exclude also the $T_{3}$ test from the analysis.

Some preliminary conclusions can be drawn from this part of the Monte Carlo:

- The $L R$, the Li and Chen $L_{T_{1} ; T_{2}}$ and Garcia's $S_{1}$ tests are not appropriate tests for changes in the weights matrix of a spatial model. They are strongly affected by the high dimensionality problem which results in unacceptable size inflation.

- Neither can we recommend Srivastava et al.'s $T_{3}$ or Srivastava and Yanagihara's $Q_{2}$ test. These tests are consistently undersized and their estimated power function is unacceptable for the case we are studying. This lack of power is not corrected by increasing (time, spatial) sample size nor after introducing higher spatial dependence.

- Bai et al's $T_{N}$ test is affected by the high dimensionality problem, requiring a concentration index no greater than 0.1. Under these conditions, the empirical size of the test appears to be approximately correct 
(in spite of slight oversizing) and its power function increases monotonically with $\mathrm{T}$, time span, and the value of the spatial autocorrelation coefficient.

- This monotonicity is maintained in the cases of the $t_{T n}$ of Schott and $T_{2}$ of Srivastava. The two tests attain good power especially in cases of high time spans. Also, their power increases quickly for cases of fixed $T$ and large $n$ especially if there is a medium to large coefficient of spatial dependence in the DGP.

- As expected, the three tests under consideration $\left(T_{N}, t_{T n}\right.$ and $\left.T_{2}\right)$ work considerably better for SLM processes, which induce global patterns with stronger symptoms of dependence. Srivastava's $T_{2}$ appears slightly superior in the case of SLM processes whereas Schott's $t_{T_{N}}$ seems preferable for dectecting breaks in local processes, like the SEM. Greater differences in the weight matrices imply higher power to detect the change as is clear by comparing results in Tables 4a,b with those of Table 4c,d.

Figures 1 to 4 show the behaviour of the three selected tests when the location of the breakpoint is not known. Each graph reproduces the average value of the corresponding statistic (vertical axis) for the hypothesis that there is a break in period $t$, in the horizontal axis. The breakpoint is located in period 75, but the user does not know that. The tests are evaluated sequentially in a rolling process initiated in period 60 and finished in period 90 (65 and 85 in Bai et al.'s $T_{N}$ test).

Figure 1 describes the case of a change from a rook type pattern to a system of weights based on the inverse of distance, in a SLM model (top panel) and in SEM model (bottom panel). Figure 2 corresponds to a softer change, from a rook pattern to a queen pattern.

Overall, the $T_{N}$ test works pretty well rejecting the null of equal covariance matrices in the whole interval inspected. The value of the test increases with the coefficient of spatial dependence but diminishes with the number of cross-sectional units (a reflection of the dimensionality problem). A Upattern emerges in cases of a high concentration index $\left(n=64\right.$ and $c_{i}=$ 0.85) combined with weak symptoms of spatial dependence. The maximum value of the sequence of $T_{N}$ tests roughly coincides with the location of the breakpoint, in period 75. As expected, the procedure works significantly worse in the case of SEM processes and small changes in the weights matrix. 


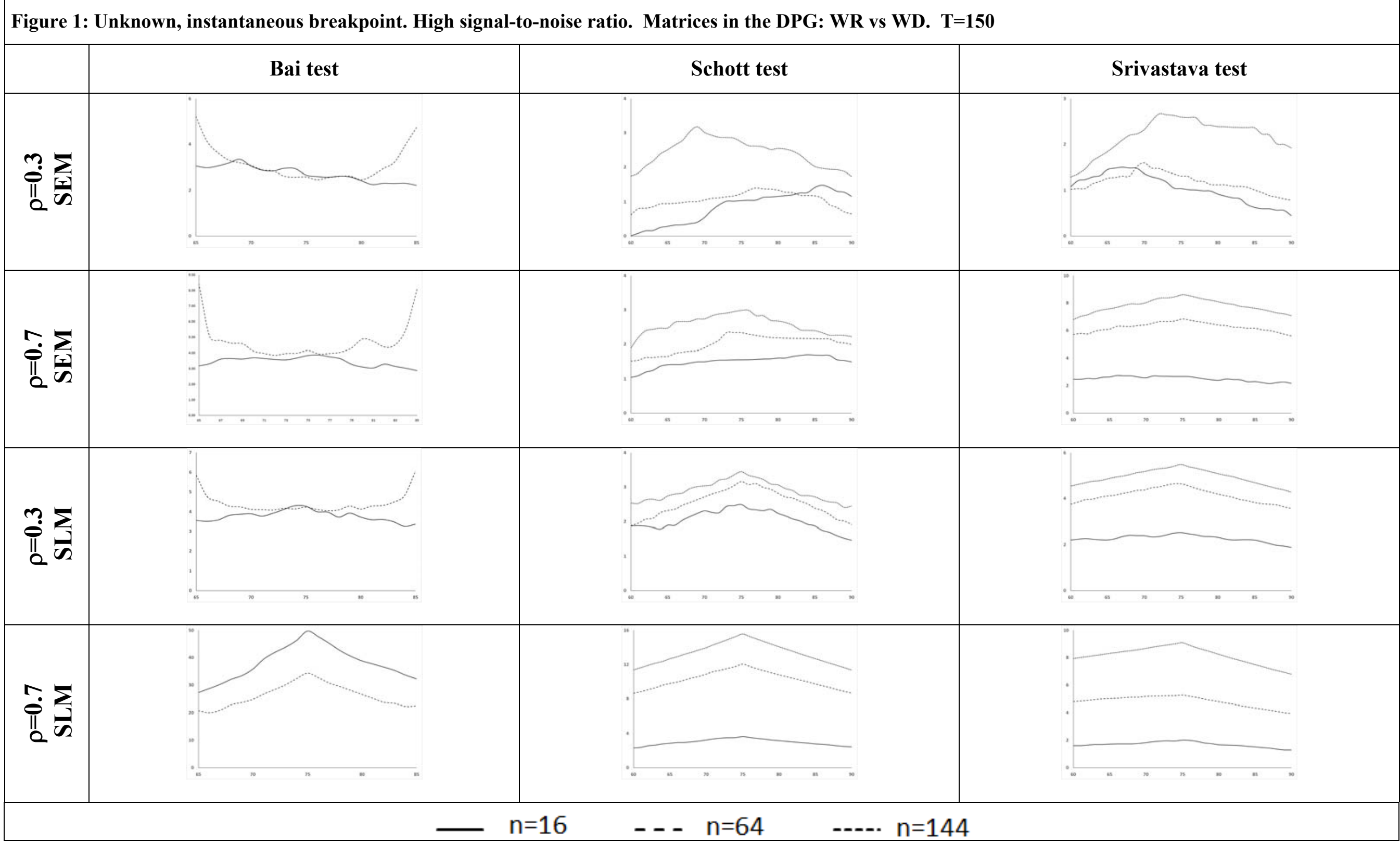




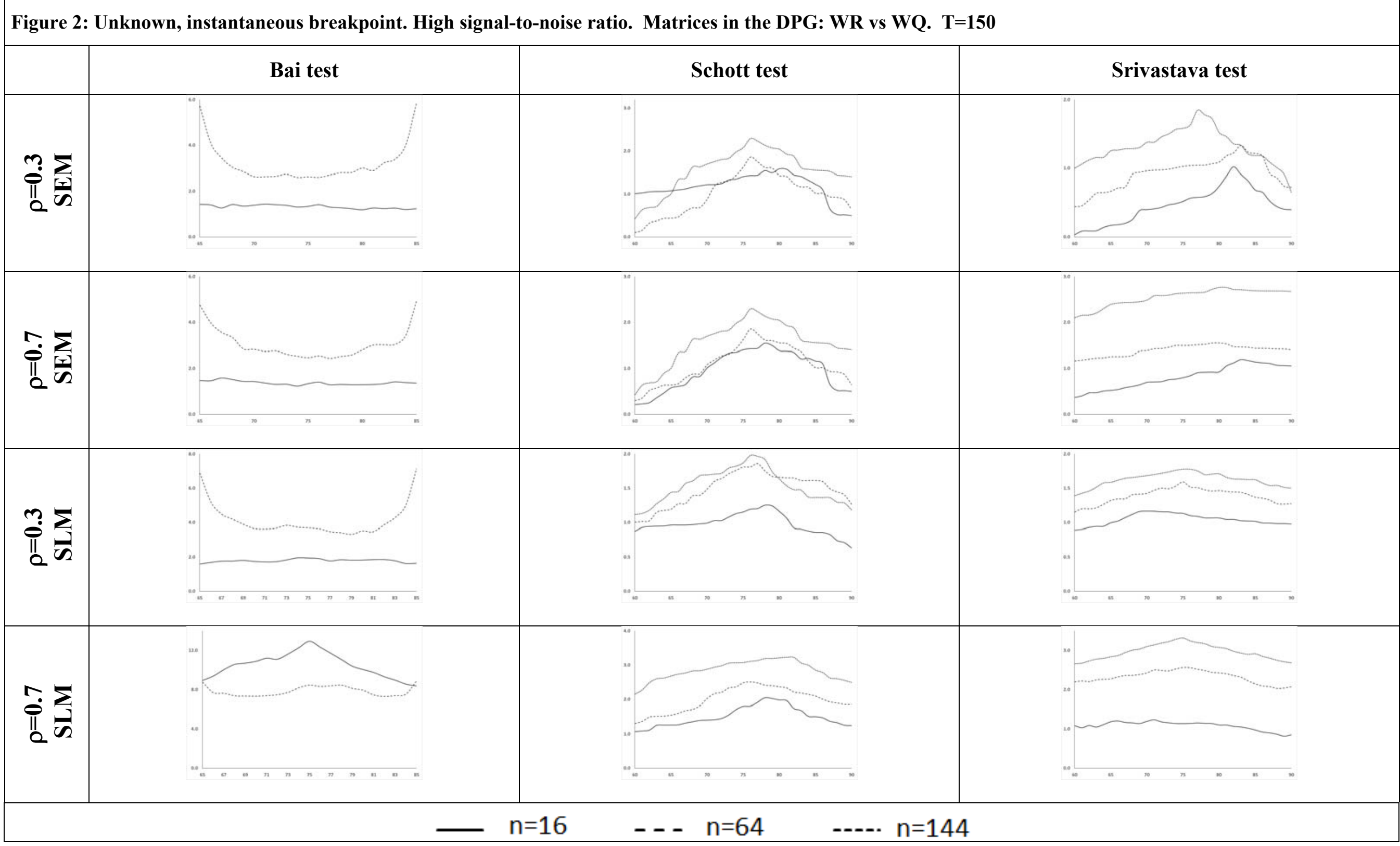




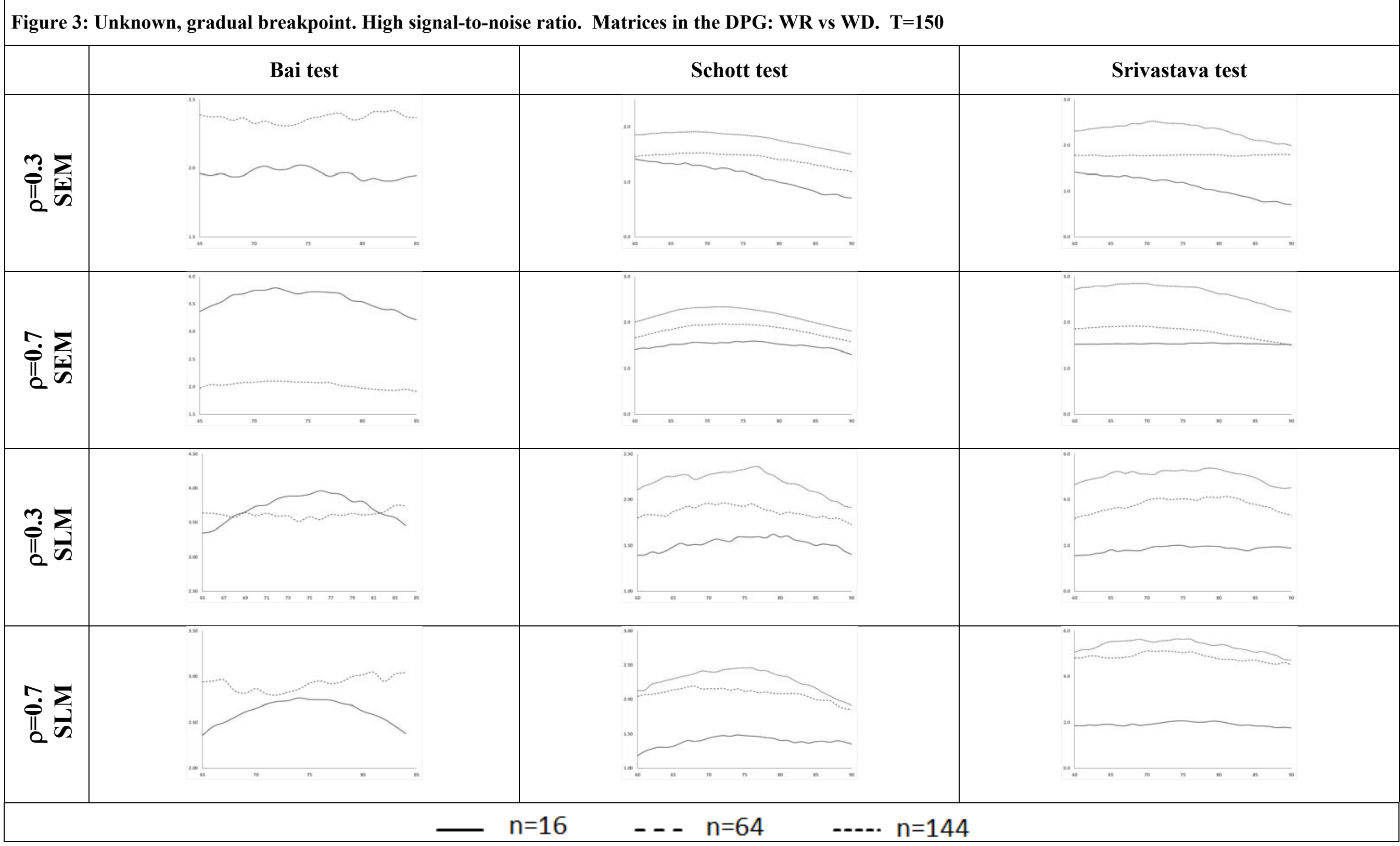


The other two tests, the $t_{T n}$ of Schott and the $T_{2}$ of Srivastava, maintain a similar profile. Both work better with changes in the weights matrix of a SLM process and where the differences between the two matrices are substantial. The identification of the location of the breakpoint is effective in the SLM case and more uncertain under SEM processes. Smaller (spatial) sample sizes, lower values in the spatial autocorrelation coefficient, local processes of dependence or soft changes in the weights matrix are factors that worsen the functioning of these tests.

The discussion is completed by reviewing Figure 3 which illustrates the case of a gradual change in the weights matrix. Now the change from one matrix to the other is not instantaneous but gradual. The transition begins in period $t=67$ and it is not fully completed until $t=82$. As before, the tests are obtained in a rolling process from observations 60 to 90 (65 to 85 for the Bai et al. test). The $T_{N}$ test works pretty well, in general, rejecting the null of equal covariance matrices in the whole testing period. The impact of the high-dimensionality problem affects the power curves of the test for the case of $n=64$, which now is very flat. The two other tests tend to work similarly well. The Schott test appears to be more sensitive to the gradual change of the weights matrix than the Srivastava test. As before, the change is more difficult to detect in the case of SEM processes and for low values of the spatial correlation coefficient. Finally, let us note the smoother curves obtained for the three tests. In all the cases, the estimated power functions are a bit more diffuse around a local peak than if the change is instantaneous; moreover, this local peak is next to the point where the transition process has completed half the total change ( $T=75$ once again).

\section{Case studies}

\subsection{Case study I: Unemployment in Spanish regions}

A distinctive feature of the Spanish economy is the high unemployment, whose spatial dimension is characterized by the persistent and remarkable differences between regions (e.g., Lopez-Bazo et al, 2005).

We can provide different explanations for such disparities. Some rely on the assumption of equilibrium, others on the notion of disequilibrium. According to Marston (1985), unemployment is a steady-state function of factor endowment. As long as they are stable through time but different 
between regions, we cannot expect rapid changes in the short run. From this perspective, unemployment is basically a local question that reflects imbalances in each regional labour market. The neoclassical paradigm posits the existence of a competitive equilibrium among the regions, where the unemployment rate will level off (Blanchard and Katz, 1992). In the short run, regional disparities result from market rigidities or mobility restrictions that should disappear in the long-run. The adjustment process will be faster or slower depending on regional circumstances, that may persist for a long time. Partridge and Rickman (1997) merge both approaches by combining disequilibrium factors (e.g., economic growth, technology) and equilibrium mechanisms, (e.g., industrial composition, wages) with demographic trends, locational amenities and institutions.

Furthermore, there is an extensive literature (e.g., Zeilstra and Elhorst, 2014) showing that unemployment rates are not only driven by intra-regional factors; extra-regional factors have also a role. Beyer and Smets (2015), from a slightly different perspective, highlight the importance of common factors in the European case (related, for example, to country effects) which strengthens the interdependence of regional unemployment figures.

The interest of the Spanish case is reinforced by the institutional framework. Franco's dictatorship ended in the 70s (Franco died in 1975). Then, the country embarked on a transition from a highly centralized system to a quasi-federal structure with 17 regions, Autonomous Communities (CCAA from now on). The set up of the so-called "Autonomic State" was finished in 1983. The decentralisation process included own-source revenues for subcentral governments and tax sharing agreements between the regions and the central government.

Actually, CCAA have substantial control over tax rates and on different legislative areas such as education (transferred between 1995 and 1999), public health (transferred in 2002), labour markets, consumers and commercial activities, etc. Key dates for the new system are the agreements of 1986, 1992, 1996, 2001 and 2009, clearly visible in Figure 4 which depicts the percentage of public expenditure under control of the regions. Currently, regional governments are responsible for $45 \%$, approximately, of total public expenditure and almost $60 \%$ of government employment.

Spanish regional unemployment combines two important features for us: it is expected to exhibit a strong cross-sectional dependence and, probably, this structure has suffered changes because of the decentralization process. We are going to study this case for the period 1980:1 to 2013:4, using quar- 
terly data.

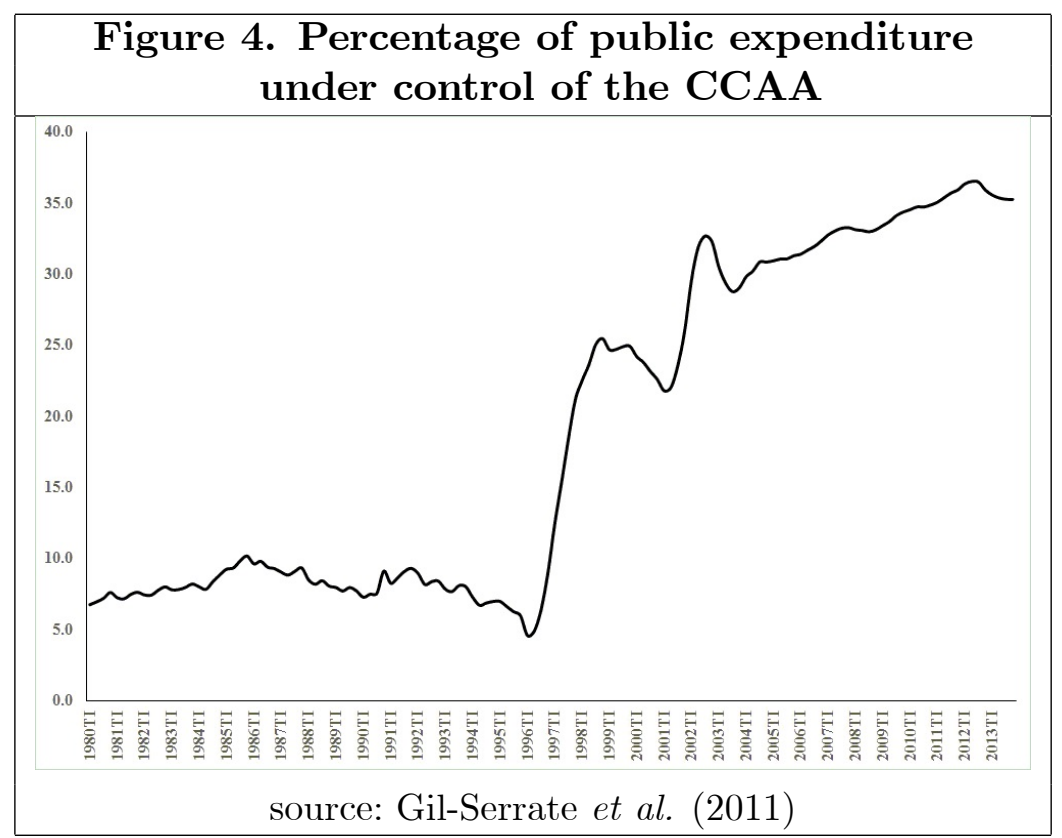

We follow the mixed approach to labour markets of Partridge and Rickman (1997) but the selection of variables has been highly constrained by data availability. A key element is unemployment by CCAA, taken from the 'Encuesta de Población Activa', Working Population Survey (Instituto Nacional de Estadística, INE, several years). Wages and economic activity in the region are the main factors driving unemployment. Quarterly Labour Cost Survey (INE, several years) is the source of the former and Spanish Regional Accounts (INE, several years) that of the second. INE produces only annual estimates of regional GDP that have been disaggregated into quarterly data according to DiFonzo (1990), using regional employment and regional industrial production indices as high-frequency indicators.

The three panel series are I(1), as indicated in Table 6, where $l p$ stands for the $\log$ of the regional gross domestic product, $l u$ is the $\log$ of regional unemployment and $l w$ the log of regional wages. $P M S B$ is the panel modified Sargan-Barghava test of Bai and $\mathrm{Ng}$ (2010) for testing non-stationarity in the idyosincratic component of the panel series, $M Q_{c}$ is the Bai and $\mathrm{Ng}$ (2004) test to determine the number of common stochastic trends in the common factors of the panel series (whose final number is indicated as $r$ in the table); CIPS* is the Pesaran (2007) test for panel unit roots; $t_{a}$, and $t_{b}$ 
are Moon and Perron (2004) tests for panel unit roots and n.c.f. indicates the number of common factors in the Moon-Perron tests as determined by the Akaike Information Criteria. All the test include individual effects and time trend. PMSB, CIPS*, $t_{a}$, and $t_{b}$ are asymptotically distributed as standard normals ( $\mathrm{p}$-value in brackets) whereas the critical values for the $M Q_{c}$ statistic appear in Table 1 of Bai and $\mathrm{Ng}$ (2004). The Moon-Perron tests cast some doubt on the nonstationarity of $l u$ and $l w$ which, however, are overwhelmingly offset by the other two tests (the Choi tests, not in the table, corroborate this conclusion).

\begin{tabular}{|c|c|c|c|c|c|c|}
\hline \multicolumn{7}{|c|}{ Table 6. Panel unit root tests } \\
\hline \multicolumn{7}{|c|}{$H_{0}: I(1)$ vs $H_{A}: I(0)$} \\
\hline & \multicolumn{2}{|c|}{$l p$} & \multicolumn{2}{|c|}{$l u$} & \multicolumn{2}{|c|}{$l w$} \\
\hline$P M S B$ & -0.4683 & $(0.3200)$ & -0.4831 & $(0.3147)$ & -0.9794 & $(0.1638)$ \\
\hline$M Q_{c}$ & -27.5893 & $r=4$ & -30.8682 & $r=3$ & -3.0300 & $r=1$ \\
\hline$C I P S^{*}$ & -2.4220 & $(0.6750)$ & -2.4931 & $(0.2750)$ & -2.1339 & $(0.8150)$ \\
\hline$t_{a}$ & 0.5300 & $(0.7019)$ & -1.7276 & $(0.0420)$ & -1.6730 & $(0.0472)$ \\
\hline$t_{b}$ & 0.4895 & $(0.6878)$ & -1.7380 & $(0.0411)$ & -0.6189 & $(0.2680)$ \\
\hline n.c.f. & \multicolumn{2}{|c|}{4} & \multicolumn{2}{|c|}{3} & \multicolumn{2}{|c|}{4} \\
\hline \multicolumn{7}{|c|}{$H_{0}: I(2)$ vs $H_{A}: I(1)$} \\
\hline & \multicolumn{2}{|c|}{$l p$} & \multicolumn{2}{|c|}{$l u$} & \multicolumn{2}{|c|}{$l w$} \\
\hline$P M S B$ & -2.3647 & $(0.0092)$ & -2.4552 & $(0.0063)$ & -3.2125 & $(0.0007)$ \\
\hline$M Q_{c}$ & -77.7600 & $r=0$ & -82.4687 & $r=0$ & -134.6321 & $r=0$ \\
\hline$C I P S^{*}$ & -4.0609 & $(0.0100)$ & -6.4003 & $(0.0100)$ & -5.1347 & $(0.0100)$ \\
\hline$t_{a}$ & -371.0119 & $(0.0000)$ & -326.4307 & $(0.0000)$ & -344.9117 & $(0.0000)$ \\
\hline$t_{b}$ & -35.6467 & $(0.0000)$ & -53.0366 & $(0.0000)$ & -61.6577 & $(0.0000)$ \\
\hline n.c.f. & \multicolumn{2}{|c|}{4} & \multicolumn{2}{|c|}{3} & \multicolumn{2}{|c|}{4} \\
\hline
\end{tabular}

Moreover, the three variables appear to be cointegrated according to Westerlund and Pedroni tests shown inTable 7. The panel statistics of Pedroni (1999) are based on pooling different estimates, from the estimated residuals, across members while the group statistics simply average these estimates. $\rho$ and $t$ statistics can be seen as variations of the analogous $\rho$ and $t$ tests of Phillips and Perron; the first is a non-parametric variance ratio statistic. The four tests are asymptotically distributed as standard normal. The variance ratio test is right-sided, while the other tests are left-sided. Similarly, the $P$ statistics of Westerlund (2007) pool information over all the cross-sectional units whereas the $G$ statistics are obtained as weighted aver- 
ages of individual estimates. They are asymptotically normally distributed; $Z$-value denotes the standardized value of the statistic. The pvalue is robust to cross-sectional dependence and has been obtained after 400 bootstraps. All the tests include individual effects and time trend. Maximum truncation lags are set to 4 and determined using data dependent criteria.

\begin{tabular}{|c|c|c|}
\hline \multicolumn{3}{|c|}{ Table 7. Panel cointegration tests } \\
\hline & PEDRONI tests \\
\hline Variance ratio & $1.938^{*}$ & Group statistics \\
\hline$\rho$ statistic & $-8.884^{*}$ & $-8.406^{*}$ \\
\hline$t$ statistic & $-8.315^{*}$ & $8.137^{*}$ \\
\hline$A D F$ statistic & -0.305 & 0.015 \\
\hline \multicolumn{2}{|c|}{ WESTERLUND tests } \\
\hline \multicolumn{2}{|c|}{ Z-value } & pvalue \\
\hline$G_{t}$ test & $-3.241^{*}$ & 0.008 \\
\hline$G_{a}$ test & 0.869 & 0.605 \\
\hline$P_{t}$ test & $-3.711^{*}$ & 0.006 \\
\hline$P_{a}$ test & -1.815 & 0.097 \\
\hline$*:$ denotes rejection of the null of no cointegration at a $5 \%$ significance level. \\
\hline
\end{tabular}

Eight of the eleven cointegration tests reject the null of no cointegration whereas the two $A D F$ tests in the case of Pedroni and the two tests based on the long run variance estimators of Westerlund detect problems. Overall, it seems the evidence supporting the assumption of cointegration is stronger.

This period has been crucial for the evolution of the contemporary Spanish economy and includes many important events such as inclusion in the European Common Market in 1986, the crisis of 1993, the full decentralization of the Public Administration or the economic crash in the second semester of 2007 and subsequent downturn. We look for structural breaks using the Banerjee and Carrión-i-Silvestre test (2015), which reveals a significant breakpoint in the second quarter of 2000 (weaker symptoms of a second break were detected also at the beginning of the 1990s and/or in 2008 , depending on the region $)^{3}$. Let us note that the procedure detects the presence of, at least, 4 non-stationary common factors, which would mean

\footnotetext{
${ }^{3}$ We do not report the results of their $t_{e}^{\tau}$ cointegration test because there is strong evidence of cross-sectional dependence in the idiosyncratic errors, distorting its asymptotic distribution.
} 
that the observed variables do not cointegrate by themselves alone (common factors are needed to obtain a significant long-run relationship). We will not go deeper into this question, which may be connected with the problems of cointegration raised in Table 7 . The model we are using, for reasons of lack of information, is an oversimplified version of the functioning of a labour market and, probably, it is somewhat misspecified. Our impression is that those nonstationary common factors are related to elements of the national or international cycle not included in the model.

Literature on labour markets agrees on the negative impact of economic activity on unemployment. The relation is more controversial in relation to wages: a positive impact is predicted from a neoclassical perspective although there are doubts from a social-democratic view. Using previous results, we approach this discussion through an Autoregressive Distributed Lag Model, $A R D L\left(p, q_{1}, q_{2}\right)$; see Pesaran et al, 2001. The lengths $p, q_{1}$ and $q_{2}$ of the $A R D L$ have been fixed paying attention to the assumption of serially uncorrelated disturbances and to the Akaike Information Criterion. Results indicate that the most adequate lag lengths, to eliminate residual serial correlation, are $p=4, q_{1}=2$ and $q_{2}=2$. The ARDL has been parameterized into an error correction equation such as the following:

$$
\left.\begin{array}{c}
\Delta l u_{i t}=\gamma_{i}\left(l u_{i t-1}-\beta_{1 i} l p_{i t}-\beta_{2 i} l w_{i t}-\beta_{3 i} d_{i t} \times l w_{i t}\right)+\alpha_{i}+\pi_{i} d_{i t} \\
\sum_{j=1}^{4} \theta_{1 j, i} \Delta l u_{i t-j}+\sum_{j=1}^{2} \theta_{2 j, i} \Delta l p_{i t-j}+\sum_{j=1}^{2} \theta_{3 j, i} \Delta l w_{i t-j}+u_{i t} ; \\
i=1, \ldots, 17 ; t=1980: 1, \ldots, 2013: 4 ; d_{i t}=1 \text { if } t \geqslant 2000: 2
\end{array}\right\}
$$

Another problem to consider is the homogeneity of the parameters of the cointegration equation among the different CCAA. A way of solving this question is by comparing the MG and PMG estimates. The first (Pesaran and Smith, 1995) relies on estimating $n$ time-series regressions, one for each CCAA, and averaging the coefficients, whereas the PMG estimator (Pesaran et al., 1999) is a mixture of pooling and averaging of coefficients. The comparison can be made through a simple Hausman test where the MG estimates are consistent under the null (homogeneous parameters) and the alternative (heterogeneous parameters) hypothesis, whereas the PMG estimates are efficient under the null but biased under the alternative. In this case, the Hausman statistic takes a value of 2.44 (3 d.o.f.) and a p-value of 0.4861 which points in favor of the mixed PMG estimates. Table 8 reports the results 
corresponding to the common panel cointegration equation (top panel) and the average of the short-run parameters (including a measure of dispersion of these estimates). Misspecification tests for temporal and cross-sectional dependence complete the information. The details of the short-run estimates of the $A R D L(4,2,2)$ corresponding to each Autonomous Community appear in Table B1 in Appendix B.

A striking result in this Table is the increased impact of regional wages after the structural break in 2000:2. The relation with unemployment was inelastic before this date ( $5 \%$ confidence interval is $0.53-1.04)$ but becomes highly elastic afterwards (the confidence interval is $2.78-3.66$ ). This result highlights the increasing importance of purely regional factors in the determination of unemployment, as a consequence of the decentralization process. Economic activity, as expected, appears as an important factor reducing regional imbalances, with a more than proportional, stable impact.

The speed of adjustment $\left(\gamma_{i}\right)$ is significant and negative in the 17 CCAA with a minimum value in the case of Navarre, -0.021 , and a maximum in La Rioja, -0.233 . The cross-equation restriction of homogeneity in this parameter is strongly rejected with a likelihood ratio test of 50.40 (pvalue of 0.0000). The short-run impact of regional economic activity on unemployment (sum of parameters $\theta_{21}$ and $\theta_{22}$ ) is negative and more than proportional; in fact, the null hypothesis of a zero short-run impact, against the alternative of a negative impact, is rejected in 14 out of the 17 regions. The situation is not so clear in the case of wages where this hypothesis cannot be rejected in almost half of the CCAA.

The $C D$ test indicates the existence of strong spatial interaction in the residual of the $A R D L(4,2,2)$ for the Spanish unemployment series. A natural reaction would be to fix the misspecification by including a SEM structure. Obvious questions are:

- what weighting matrix?

- is it in fact the same for the three decades? 


\begin{tabular}{|c|c|c|}
\hline \multicolumn{3}{|c|}{$\begin{array}{l}\text { Table 8. Estimation of the } \operatorname{ARDL}(4,2,2) \\
\text { for the unemployment case }(18)\end{array}$} \\
\hline \multicolumn{3}{|c|}{ Long run coefficients } \\
\hline & Estimate & $\mathrm{p}$-value \\
\hline$\beta_{1}(\operatorname{gdp})$ & 1.6324 & 0.0000 \\
\hline$\beta_{2}$ (wages) & 0.7896 & 0.0000 \\
\hline$\beta_{3}$ (wages $\times$ time dummy) & 2.4287 & 0.0000 \\
\hline \multicolumn{3}{|c|}{ Short run coefficients } \\
\hline & Mean estimate & stand. dev. \\
\hline$\gamma_{i}$ & -0.0891 & 0.0334 \\
\hline$\alpha_{i}$ & 1.5823 & 0.0226 \\
\hline$\pi_{i}$ & -0.5105 & 0.0386 \\
\hline$\theta_{11}$ & 0.1656 & 0.1901 \\
\hline$\theta_{12}$ & -0.2963 & 0.1842 \\
\hline$\theta_{13}$ & 0.3348 & 0.1419 \\
\hline$\theta_{14}$ & -0.0669 & 0.3400 \\
\hline$\theta_{21}$ & -1.9912 & 0.0774 \\
\hline$\theta_{22}$ & 0.1592 & 0.4133 \\
\hline$\theta_{31}$ & -0.1144 & 0.3057 \\
\hline$\theta_{32}$ & -0.0331 & 0.3640 \\
\hline Serial correlation & Estimate & $\mathrm{p}$-value \\
\hline$r_{1}$ & -0.1114 & 0.1738 \\
\hline$r_{2}$ & 0.0206 & 0.3878 \\
\hline$r_{3}$ & 0.0166 & 0.3916 \\
\hline$r_{4}$ & -0.0400 & 0.3584 \\
\hline$r_{5}$ & 0.0011 & 0.3989 \\
\hline Cross-sect correl. & Estimate & p-value \\
\hline CD test & 37.2447 & 0.0000 \\
\hline \multicolumn{3}{|c|}{$\begin{array}{l}\text { Note: } r_{j} \text { denotes the mean correlation coefficient between the cross-sections residuals } \\
\text { separated by j periods. Given that } \mathrm{T} \text { is larger than } \mathrm{N} \text {, it is compared with a } \mathrm{N}(0,1) \text {. } \\
C D: \text { denotes Pesaran's test of cross-sectional dependence in panel data. } \\
\text { See Arellano and Bond (1991) and Pesaran (2004), respectively. }\end{array}$} \\
\hline
\end{tabular}

Different hypotheses can be formulated in relation to the first question, none of them definitive. The nonparametric procedure of Bhattacharjee and Jensen-Butler (2013) produces the weighting matrix listed in Table B2 in Appendix B. The answer to the second question is negative: this matrix can- 
not be taken as constant for the three decades. Figure 5 shows the rolling estimates of the $T_{N}$ test of Bai et al (2009) and of the $t_{T n}$ test of Schott (2007). The search span contains $60 \%$ of the observations (that is, 80 quarters) and it is centered in the middle of the sample (1998:1). Both tests peak in the second semester of 2000, where the Banerjee and Carrión-i-Silvestre procedure identifies a similar break in the cointegration equation. Notice that this date roughly coincides with the completion of the transference of the second large set of competencies to the regions. We do not have a clear cut explanation for the sharp fall produced in both tests after the peak.

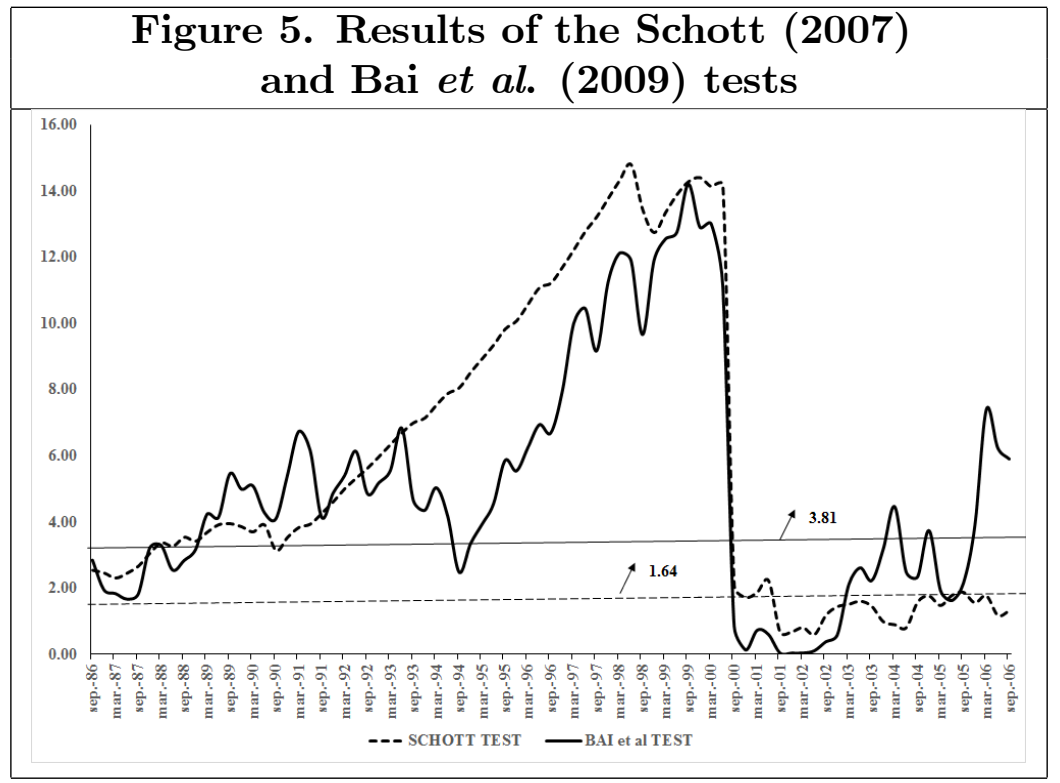

Under the alternative hypothesis there are, at least, two weighting matrices working in the sample, as they appear in Tables B3 and B4 in Appendix B. Once more, the matrices have been estimated using the nonparametric algorithm of Bhattacharjee and Jensen-Butler (2013).

Some comments are in order here. First, the $\mathbf{W}$ matrix obtained for the first period is sparser than that for the second. This is consistent with the decentralization hypothesis: as the regions gain control over their own decisions, the interrelation between them increases. Following this change, there has been a realignment among the regions in terms of 'good' and 'bad' neighbours. During the first period, the ranking of regions with a positive impact on the others is headed by geographically central regions, such as CastilleLeon, Castille-La Mancha or Madrid; in the second period, the group of 
regions with positive influence has moved towards the Mediterranean axis (both Castilles, Catalonia, Valencian Community are there). On the contrary, peripheral regions appear at the top of the ranking of regions with negative impulses in the first period (Balearic Islands, Cantabria, Murcia), which is made by Northern and peripheral regions in the second (Asturias, Cantabria, Canary Islands, Extremadura). This picture is also consistent with the gradual but firm displacement of the centre of gravity of the Spanish economy from the North-West to the South-East of the peninsula. Another interesting result is the 'lack of geography' in these matrices whose relation with the concept of contiguity is very feeble, if not entirely absent. This also accords with the distinction between micro and macro data in the sense that aggregating spatial data leads to the weakening of purely geographical

relations, favouring other types of comovements such as national common factors.

\subsection{Case Study II: Housing prices in Spanish munici- palities}

The second example refers to housing prices in Spanish municipalities. National housing prices rose strongly in the expansion cycle that preceded the global downturn of 2007; this increment was one of the largest in Europe. However the crisis led to severe corrections in the sector, stronger than the European mean. The boom period started, approximately, in 1995 and the housing stock increased by 50 percent from 1995 to 2007, with an average of more than half a million houses built per year. Meanwhile the population increased by 6.5 million people (18\%) and the GDP per capita by more than $4 \%$ on average. The market collapsed after the financial crash of 2007 with cuts in prices by more than $30 \%$ on average (see Figure 6), GDP per capita decreased around $2.3 \%$ per year in the period 2008 to 2014 and there was an out-migration of almost half a million people.

Some factors are specific to the Spanish case, such as the massive affluence of baby-boomers in the early 2000s increasing the group of young working adults, prone to demand housing services. The loosening of financial conditions in the years before the crisis facilitated the access to credit, which spurred the demand for housing in a market with a strong preference for ownership. Moreover, fiscal policy favoured ownership, with subsidies, deductions in personal income taxes and several incentives to reinvestment 
that were partially removed from the Spanish tax system in 2012 .

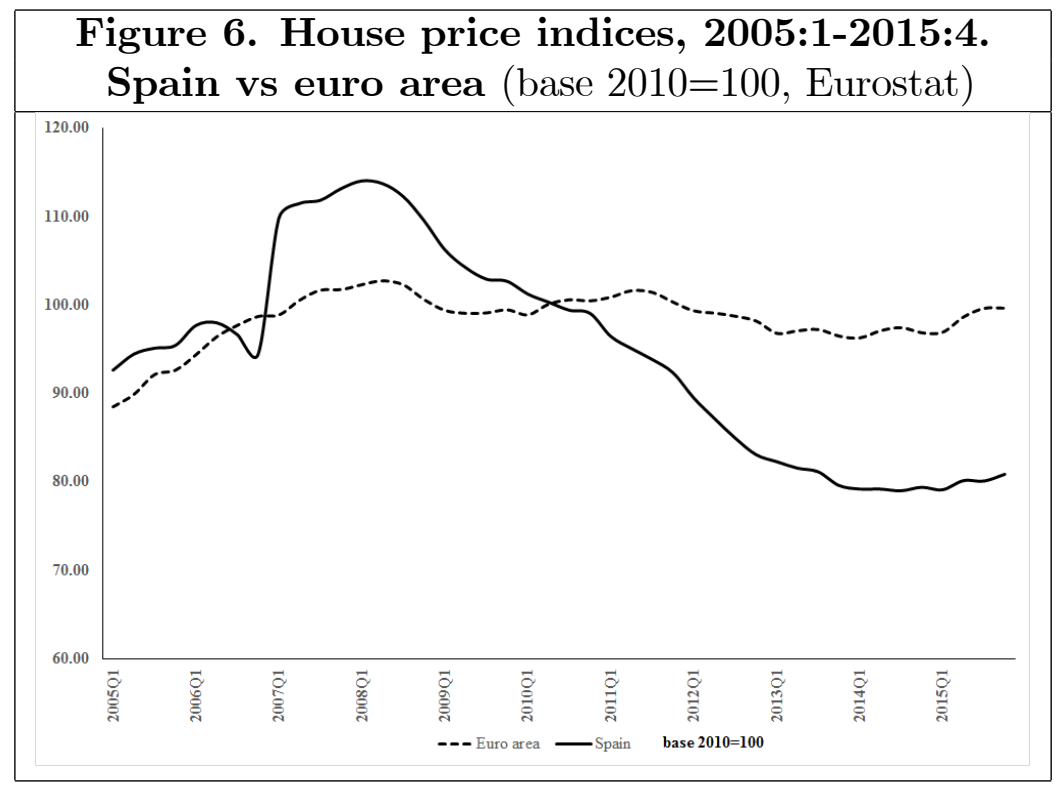

There is a rich literature devoted to real estate markets. A common starting point is the notion of real housing user cost of capital (Cameron et al., 2006, or Holly et al., 2010), which confronts the consumer with a utility maximization problem in an inter-temporal model of consumption, with two different goods: a composite consumption good and housing services. Real housing user cost of capital results from the first order conditions of the optimization problem in which interest rate, housing stock, income and demographic factors are key variables driving the formation of prices. In addition, real estate markets tend to be segmented into several sub-markets (owner-occupied houses, second residences, tourist activities, investors' property developments, etc.), each one of them with a strong spatial dynamic (e.g., Can, 1990 and 1992; Brady, 2011; Martinez and Maza, 2003, for the Spanish case).

Our case study refers to housing prices for the 284 Spanish municipalities with more than 25,000 residents (Figure 7 shows their location). The data emanate from the Spanish Department of Housing (Dirección General de Arquitectura, Vivienda y Suelo, Ministerio de Fomento), and comprise quarterly data from 2005:1 to 2015:4. However, we are not going into the direction of modelling because there is an acute problem of data unavailability at a municipal level in Spain. We merely test for structural breaks in the 
cross-sectional connections among the municipalities.

The cross-sectional dimension of this dataset, 284 municipalities, is 6 times greater than its time dimension, 44 quarters in the sample. There is a strong dimensionality problem that prevents the use of LR based tests. Moreover, given the short time dimension, it is not possible to estimate the underlying weighting matrix. As previously remarked, at most we can speculate about its stability.

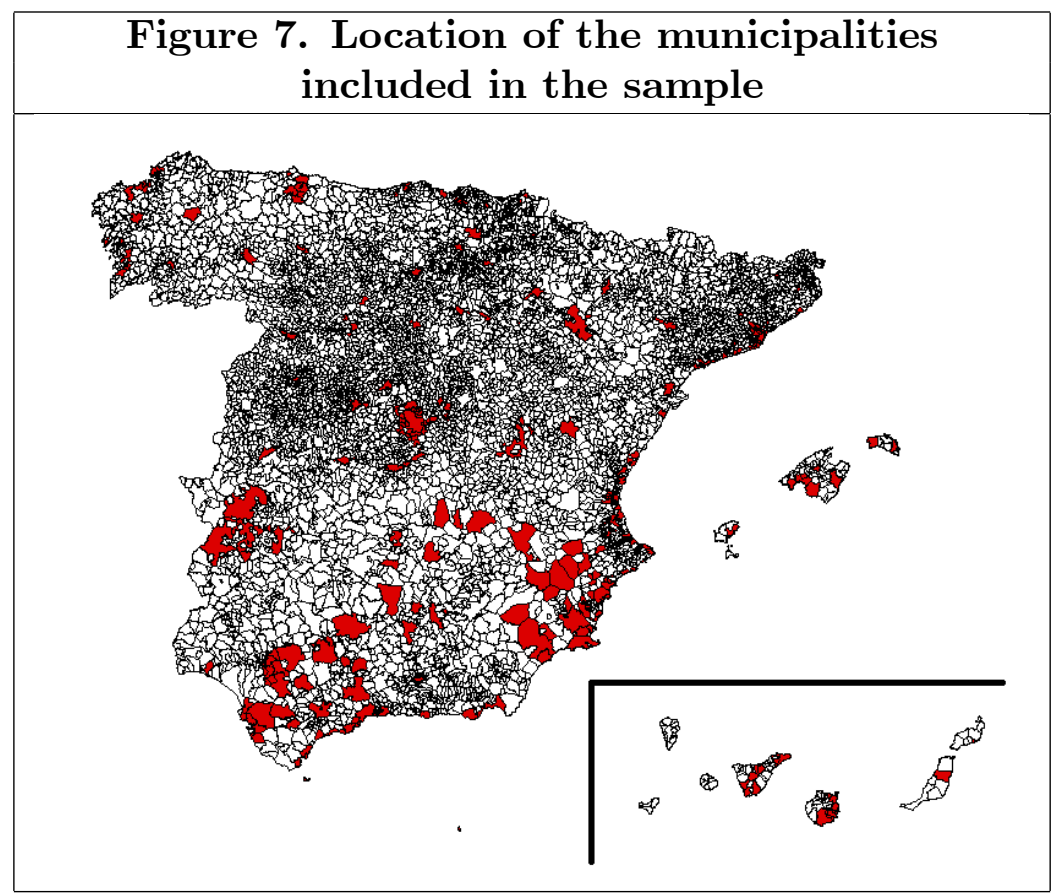

Figure 8 shows the rolling estimates of the $t_{T n}$ test of Schott and of the $T_{2}$ test of Srivastava. As before, the search span contains $60 \%$ of the observations (that is, 26 quarters) and it is centered in the middle of the sample (2010:2). The two tests offer a very similar view of this case: there is a wide time interval where they detect a break in the covariance matrix: from the beginning of 2008 to the second semester of 2011, with a peak in the first quarter of 2010. This period coincides with the outbreak of the crash and the bursting of the Spanish housing bubble.

Of course, this break may be due to other causes different from the hypothesized change in the weighting matrix (for example, changes of agents' 
expectations, in the perception of risk, etc.), but the instability in the crosssectional structure of the markets is a potential disturbing factor that cannot be neglected. This is consistent with the realignment of the Spanish real estate sector after the crash of 2007. Actually, the sector is working at a very moderate level of activity and it is highly dependent on the demand for residential housing, in very specific and well chosen locations. Real estate markets evolve at a lower scale than before and the activity is a bit more diffuse on the territory; rehabilitation and restoration of properties occupy a more prominent role to the detriment of purely touristic sub-markets. In sum, the sector appears to have acquired a more balanced spatial distribution which may have caused a break in the connections captured by the weights matrix connecting local markets.

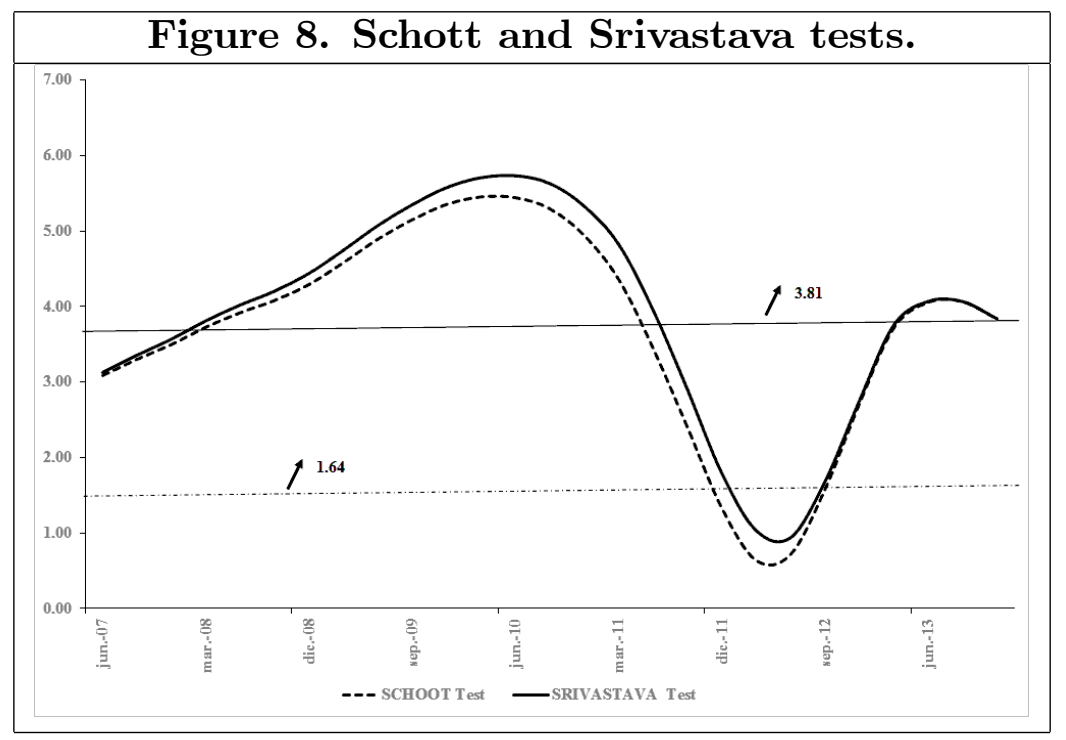

\section{Conclusion}

In recent years there has been a growing interest in different questions related to $\mathbf{W}$. There appears to be a general consensus in the sense that it is not enough to build the weights matrix exogenously according to some unspecified prior knowledge. On the contrary, the author should make explicit his/her knowledge in order that the results of the estimated models may be interpreted. Usually, one has several candidate matrices, which amounts to a decision problem that should be solved using some of the different algorithms 
that exist in the literature. In other cases, assuming that we have panel data, the $\mathbf{W}$ matrix can be estimated from the data.

Our contribution focuses on the (implicit) assumption of time constancy of $\mathbf{W}$ in a panel data framework. If the time span is large or there have been external shocks, it seems reasonable to expect changes also in the crosssectional connections. The approach in this paper consists in testing the existence of possible breaks in $\mathbf{W}$ through the corresponding covariance matrix. The literature devoted to the topic of comparing covariance matrices is large and heterogeneous, from which we have chosen a set of potentially useful tests.

The Monte Carlo experiment reported in the paper leads us to a clear conclusion: the $T_{N}$ test of Bai et al (2009), the $t_{T_{n}}$ test of Schott (2007) and the $T_{2}$ test of Srivastava (2007) are candidates that adapt well to our case. The first test tends to be slightly oversized, especially for small time sample sizes. The $T_{2}$ test is consistently undersized whereas the $t_{T_{n}}$ test of Schott is more balanced in relation to size. The estimated power function of the three tests improves very quickly, both with the time span and the spatial coefficient. The tests work quite well in the case where the location of the breakpoint is unknown and also when the change in the weight matrix is gradual. On the negative side, because the $T_{N}$ test is a corrected version of a standard Likelihood Ratio, it needs a concentration $c_{i}$ index in each subsample lower than 1. This limitation does not apply for the other two tests, built around the notion of the Frobenius norm. Their behavior is worse for processes of local dependence, such as the SEM, where the symptoms of spatial dependence are weaker. Moreover, the distance between the weight matrices involved in the change is a crucial factor to guarantee a proper functioning of the tests.

As said in the Introduction, this paper is part of ongoing research whose objective is to endogenize fully the building of the weighting matrix in a spatial model. Tests for its stability are a small step forward, and need to be combined with more flexible estimation algorithms. The potential usefulness of these techniques, combined with other tools recently developed in relation to the weights matrix has been illustrated by means of two case studies coming from the contemporary Spanish economy. Overall, the results of our approach corroborate the dominant position with respect to this economy. 


\section{Appendix A: The SEM case in the errors of a linear model}

This Appendix describes the results obtained for an extension of the Monte Carlo discussed in Section 4. Here we treat the case where the user knows that the spatial structure appears only in the error terms of the equation. The DGP simulated corresponds to expressions (16), null hypothesis, and (17), alternative hypothesis. This (partial) knowledge of the DGP allows the user to (i) estimate the equation of the mean using a consistent estimator (LS in our Monte Carlo), (ii) obtain the corresponding residuals and (iii) solve the test for breaks using the covariance matrix of the residuals from step (ii). We expect that this additional knowledge in the experiment improves the behaviour of the tests. Main results, for the $T_{N}$ test of Bai et al (2009), the $t_{T_{n}}$ test of Schott (2007) and the $T_{2}$ test of Srivastava (2007) appear in Tables A1 and A2 below, for the case of a known breakpoint, and in Figure A1, for the case of an unknown instantaneous breakpoint (results for the case of a gradual change in the weight matrix are much in line with those shown below; details are available from the authors upon request).

This partial knowledge is highly beneficial for the three tests, both in power and in size. The $T_{N}$ test appears to be, still, slightly oversized especially for high values of $c_{i}$, the concentration index. The $T_{2}$ of Srivastava continues to be undersized although the intensity of the spatial dependency tends to correct this deficit. The size of the $t_{T_{n}}$ test of Schott is right for all the cases in the experiment. The impact on the estimated power of the three tests is clear. Overall, data on power have increased around 30\%-40\% in relation to the situation described in Tables $4 \mathrm{a}-4 \mathrm{~d}$. The power of the tests improves with the sample size (in the cross-section and/or in the time dimension), with the strength of the spatial dependence and with the distance between the matrices in the break. Figure A1 confirms this amelioration for the case of an unkown instantaneous break. The U-shaped pattern is still present in the estimated power function of the $T_{N}$ test for low values of the spatial dependence coefficient. The behaviour of the other two tests is more efficient now in the sense that the peak of the sequence of values is better defined in the proximity of the (unknown) breakpoint. 


\begin{tabular}{|c|c|c|c|c|c|c|c|c|c|c|c|c|}
\hline \multicolumn{13}{|c|}{$\begin{array}{c}\text { Table A1: Estimated size (significance level=0.05). Known breakpoint. SEM in the errors of a linear } \\
\text { model. High signal-to-noise ratio. Matrix in the DGP: WR. }\end{array}$} \\
\hline \multicolumn{13}{|c|}{$\rho=0.3$} \\
\hline $\mathbf{T}$ & \multicolumn{3}{|c|}{30} & \multicolumn{3}{|c|}{60} & \multicolumn{3}{|c|}{150} & \multicolumn{3}{|c|}{250} \\
\hline $\mathrm{n}$ & 16 & 144 & 225 & 16 & 144 & 225 & 16 & $144 *$ & 225 & 16 & $144 *$ & 225 \\
\hline $\mathbf{T}_{\mathbf{N}}$ & - & - & - & 0.09 & - & - & 0.06 & 0.11 & - & 0.05 & 0.12 & - \\
\hline $\mathbf{t}_{\mathbf{T n}}$ & 0.04 & 0.04 & 0.05 & 0.04 & 0.04 & 0.06 & 0.05 & 0.05 & 0.04 & 0.06 & 0.06 & 0.07 \\
\hline $\mathbf{T}_{2}$ & 0.02 & 0.02 & 0.03 & 0.01 & 0.01 & 0.01 & 0.03 & 0.01 & 0.01 & 0.02 & 0.01 & 0.02 \\
\hline \multicolumn{13}{|c|}{$\rho=0.7$} \\
\hline $\mathbf{T}$ & \multicolumn{3}{|c|}{30} & \multicolumn{3}{|c|}{60} & \multicolumn{3}{|c|}{150} & \multicolumn{3}{|c|}{250} \\
\hline $\mathrm{n}$ & 16 & 144 & 225 & 16 & 144 & 225 & 16 & $144^{*}$ & 225 & 16 & $144^{*}$ & 225 \\
\hline$T_{N}$ & - & - & - & 0.14 & - & - & 0.16 & 0.15 & - & 0.10 & 0.06 & - \\
\hline $\mathbf{t}_{\mathbf{T n}}$ & 0.04 & 0.04 & 0.05 & 0.05 & 0.06 & 0.05 & 0.05 & 0.03 & $0.05-$ & 0.06 & 0.06 & 0.05 \\
\hline $\mathbf{T}_{2}$ & 0.04 & 0.03 & 0.03 & 0.04 & 0.04 & 0.04 & 0.06 & 0.05 & 0.05 & 0.07 & 0.05 & 0.05 \\
\hline
\end{tabular}

\begin{tabular}{|c|c|c|c|c|c|c|c|c|c|c|c|c|}
\hline \multicolumn{13}{|c|}{$\begin{array}{c}\text { Table A2: Estimated power (significance level=0.05). Known breakpoint. SEM in the error of a } \\
\text { linear model. High signal-to-noise ratio. }\end{array}$} \\
\hline \multicolumn{13}{|c|}{$\rho=0.3$ Matrices in the DGP: WR vs WD } \\
\hline $\mathbf{T}$ & \multicolumn{3}{|c|}{30} & \multicolumn{3}{|c|}{60} & \multicolumn{3}{|c|}{150} & \multicolumn{3}{|c|}{250} \\
\hline $\mathbf{n}$ & 16 & 144 & 225 & 16 & 144 & 225 & 16 & $144 *$ & 225 & 16 & $144 *$ & 225 \\
\hline $\mathbf{T}_{\mathbf{N}}$ & - & - & - & 0.46 & - & - & 0.51 & 0.72 & - & 0.64 & 0.88 & - \\
\hline $\mathbf{t}_{\mathbf{T n}}$ & 0.32 & 0.39 & 0.43 & 0.41 & 0.64 & 0.72 & 0.55 & 0.80 & 0.89 & 0.73 & 0.82 & 0.93 \\
\hline $\mathbf{T}_{2}$ & 0.28 & 0.29 & 0.44 & 0.45 & 0.49 & 0.76 & 0.58 & 0.67 & 0.80 & 0.63 & 0.75 & 0.86 \\
\hline \multicolumn{13}{|c|}{$\rho=0.7$ Matrices in the DGP: WR vs WD } \\
\hline $\mathbf{T}$ & \multicolumn{3}{|c|}{30} & \multicolumn{3}{|c|}{60} & \multicolumn{3}{|c|}{150} & \multicolumn{3}{|c|}{250} \\
\hline n & 16 & 144 & 225 & 16 & 144 & 225 & 16 & $144 *$ & 225 & 16 & $144^{*}$ & 225 \\
\hline $\mathbf{T}_{\mathbf{N}}$ & - & - & - & 0.78 & - & - & 0.83 & 0.82 & - & 0.91 & 0.89 & - \\
\hline $\mathbf{t}_{\mathbf{T n}}$ & 0.38 & 0.45 & 0.64 & 0.47 & 0.74 & 0.85 & 0.69 & 0.87 & 0.95 & 0.87 & 1.00 & 1.00 \\
\hline $\mathbf{T}_{2}$ & 0.40 & 0.46 & 0.53 & 0.52 & 0.68 & 0.78 & 0.66 & 0.78 & 0.89 & 0.79 & 1.00 & 1.00 \\
\hline \multicolumn{13}{|c|}{$\rho=0.3$ Matrices in the DGP: WR vs WQ } \\
\hline $\mathbf{T}$ & \multicolumn{3}{|c|}{30} & \multicolumn{3}{|c|}{60} & \multicolumn{3}{|c|}{150} & \multicolumn{3}{|c|}{250} \\
\hline n & 16 & 144 & 225 & 16 & 144 & 225 & 16 & $144 *$ & 225 & 16 & $144 *$ & 225 \\
\hline $\mathbf{T}_{\mathrm{N}}$ & - & - & - & 0.28 & - & - & 0.41 & 0.52 & - & 0.54 & 0.58 & - \\
\hline $\mathbf{t}_{\mathbf{T n}}$ & 0.19 & 0.26 & 0.38 & 0.32 & 0.47 & 0.61 & 0.41 & 0.60 & 0.66 & 0.63 & 0.82 & 0.93 \\
\hline $\mathbf{T}_{2}$ & 0.17 & 0.22 & 0.42 & 0.35 & 0.39 & 0.63 & 0.45 & 0.57 & 0.70 & 0.58 & 0.65 & 0.79 \\
\hline \multicolumn{13}{|c|}{$\rho=0.7$ Matrices in the DGP: WR vs WQ } \\
\hline $\mathbf{T}$ & \multicolumn{3}{|c|}{30} & \multicolumn{3}{|c|}{60} & \multicolumn{3}{|c|}{150} & \multicolumn{3}{|c|}{250} \\
\hline $\mathbf{n}$ & 16 & 144 & 225 & 16 & 144 & 225 & 16 & 144* & 225 & 16 & $144 *$ & 225 \\
\hline $\mathbf{T}_{\mathbf{N}}$ & - & - & - & 0.42 & - & - & 0.53 & 0.59 & - & 0.82 & 0.71 & - \\
\hline $\mathbf{t}_{\mathbf{T n}}$ & 0.31 & 0.35 & 0.51 & 0.37 & 0.61 & 0.75 & 0.51 & 0.66 & 0.78 & 0.73 & 0.81 & 1.00 \\
\hline $\mathbf{T}_{2}$ & 0.37 & 0.42 & 0.55 & 0.42 & 0.51 & 0.68 & 0.56 & 0.69 & 0.80 & 0.75 & 0.98 & 1.00 \\
\hline
\end{tabular}




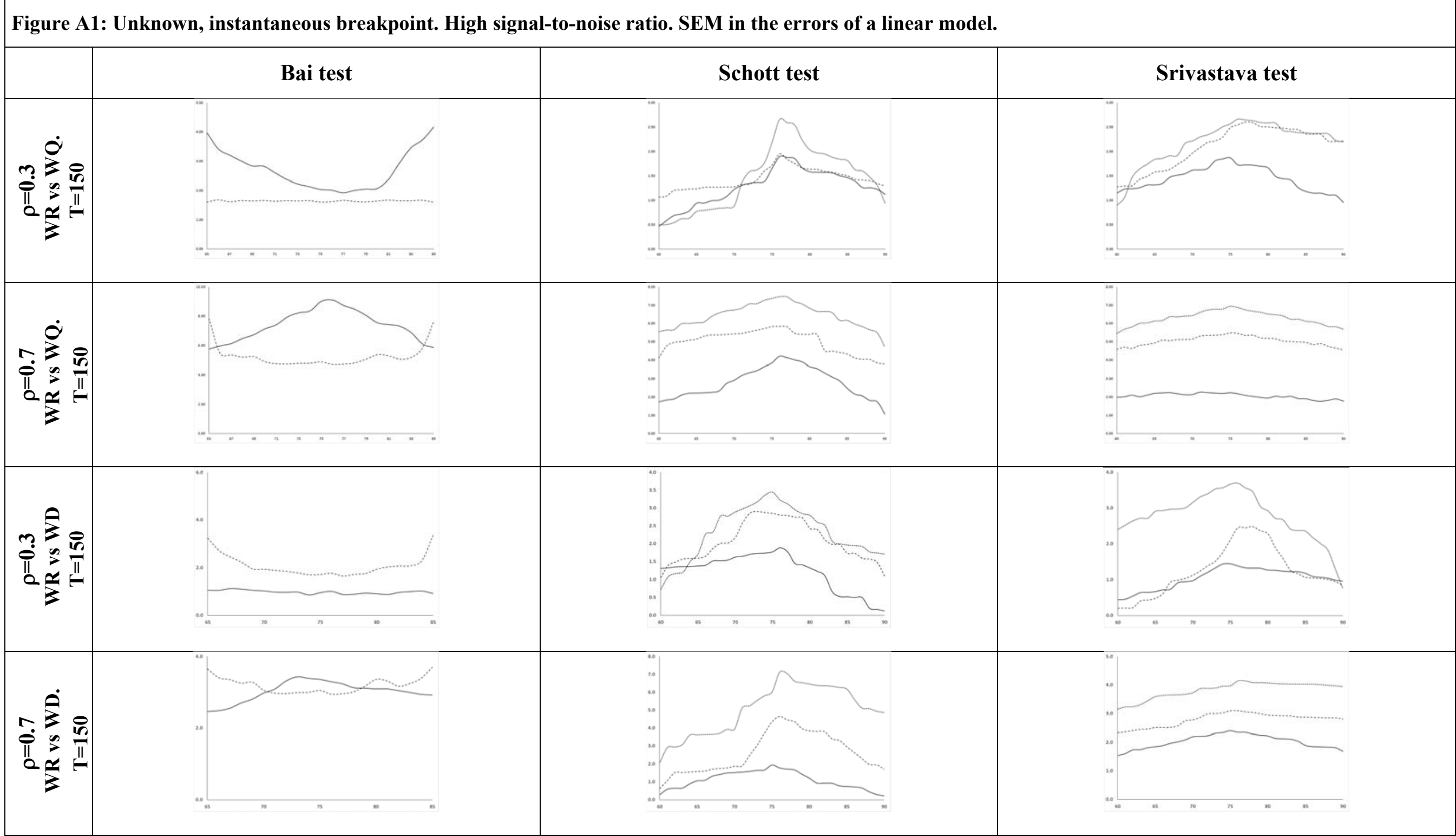


APPENDIX B: Additional results for the case of Spanish Regional Unemployment.

\begin{tabular}{|c|c|c|c|c|c|c|c|c|c|c|c|c|c|c|c|c|c|c|}
\hline \multicolumn{19}{|c|}{ Table B1: Detailed results for the ARDL $(4,2,2)$ short-run estimates } \\
\hline & \multicolumn{2}{|c|}{ ANDA } & \multicolumn{2}{|c|}{ ARAG } & \multicolumn{2}{|c|}{ ASTU } & \multicolumn{2}{|c|}{ BALE } & \multicolumn{2}{|c|}{ CANA } & \multicolumn{2}{|c|}{ CANT } & \multicolumn{2}{|c|}{ CLEO } & \multicolumn{2}{|c|}{ CMAN } & \multicolumn{2}{|c|}{ CATA } \\
\hline & Estima. & pvalue & Estima. & pvalue & Estima. & pvalue & Estima. & pvalue & Estima. & pvalue & Estima. & pvalue & Estima. & pvalue & Estima. & pvalue & Estima. & pvalue \\
\hline$\gamma_{i}$ & -0.054 & 0.001 & -0.095 & 0.002 & -0.197 & 0.000 & -0.142 & 0.001 & \begin{tabular}{|l|}
-0.087 \\
\end{tabular} & 0.001 & -0.096 & 0.002 & -0.091 & 0.000 & -0.114 & 0.000 & -0.056 & 0.005 \\
\hline$\alpha_{i}$ & 1.184 & 0.002 & 1.597 & 0.004 & 3.238 & 0.000 & 2.299 & 0.002 & 1.573 & 0.002 & 1.413 & 0.006 & 1.707 & 0.000 & 1.987 & 0.000 & 1.228 & 0.009 \\
\hline$\pi_{i}$ & -0.305 & 0.001 & -0.564 & 0.003 & -1.256 & 0.000 & -0.742 & 0.001 & -0.443 & 0.001 & -0.567 & 0.002 & -0.550 & 0.000 & -0.599 & 0.000 & -0.348 & 0.006 \\
\hline$\theta_{11}$ & 0.316 & 0.023 & 0.316 & 0.060 & 0.097 & 0.323 & -0.008 & 0.399 & 0.143 & 0.249 & 0.026 & 0.394 & 0.336 & 0.020 & 0.420 & 0.002 & 0.426 & 0.001 \\
\hline$\theta_{12}$ & -0.404 & 0.116 & -0.729 & 0.019 & -0.175 & 0.331 & -0.425 & 0.171 & -0.246 & 0.271 & -0.352 & 0.206 & -0.749 & 0.006 & -0.764 & 0.004 & -0.478 & 0.054 \\
\hline$\theta_{13}$ & 0.477 & 0.051 & 0.756 & 0.003 & -0.020 & 0.398 & 0.679 & 0.011 & 0.219 & 0.264 & 0.469 & 0.071 & 0.798 & 0.001 & 0.597 & 0.009 & 0.345 & 0.118 \\
\hline$\theta_{14}$ & -0.173 & 0.040 & -0.225 & 0.006 & 0.051 & 0.321 & -0.225 & 0.005 & -0.042 & 0.347 & -0.164 & 0.048 & -0.247 & 0.002 & -0.144 & 0.052 & -0.114 & 0.128 \\
\hline$\theta_{21}$ & -1.418 & 0.018 & -2.906 & 0.007 & -1.334 & 0.163 & -4.870 & 0.000 & -2.165 & 0.008 & -2.066 & 0.048 & -1.061 & 0.139 & -1.372 & 0.013 & -2.864 & 0.001 \\
\hline$\theta_{22}$ & -0.448 & 0.247 & 0.718 & 0.249 & 1.020 & 0.099 & 1.846 & 0.075 & -0.073 & 0.396 & 0.445 & 0.333 & -0.144 & 0.379 & -0.462 & 0.171 & 0.242 & 0.367 \\
\hline$\theta_{31}$ & 0.136 & 0.109 & -0.245 & 0.069 & -0.748 & 0.000 & 0.121 & 0.365 & -0.104 & 0.292 & -0.151 & 0.165 & -0.090 & 0.197 & -0.081 & 0.350 & -0.026 & 0.380 \\
\hline$\theta_{31}$ & -0.075 & 0.115 & 0.026 & 0.374 & 0.174 & 0.113 & -0.207 & 0.154 & -0.017 & 0.389 & -0.012 & 0.393 & -0.055 & 0.194 & 0.017 & 0.390 & -0.127 & 0.017 \\
\hline
\end{tabular}

\begin{tabular}{|c|c|c|c|c|c|c|c|c|c|c|c|c|c|c|c|c|}
\hline & \multicolumn{2}{|c|}{ CVAL } & \multicolumn{2}{|c|}{ EXTR } & \multicolumn{2}{|c|}{ GALI } & \multicolumn{2}{|c|}{ MADR } & \multicolumn{2}{|c|}{ MURC } & \multicolumn{2}{|c|}{ NAVA } & \multicolumn{2}{|c|}{ PAVA } & \multicolumn{2}{|c|}{ RIOJ } \\
\hline & stima. & pvalue & tima. & pvalue & tima. & pvalue & Estima. & value & tima. & pvalue & stima. & pvalue & stima. & pvalue & istima. & pvalue \\
\hline$\gamma_{i}$ & -0.105 & 00 & 60 & 00 & 084 & & 89 & 01 & -0.083 & 1 & -0.021 & 337 & 0.040 & 12 & .233 & 000 \\
\hline$\alpha_{i}$ & 2.132 & 0.001 & 2.607 & 000 & 588 & 001 & 873 & 02 & 91 & 0.002 & 318 & 333 & .765 & .016 & 3.015 & 0.000 \\
\hline$\pi_{i}$ & .572 & 0.000 & 891 & 00 & 479 & 00 & 43 & 02 & 433 & 01 & 100 & 364 & 286 & 010 & .336 & 0.000 \\
\hline$\theta_{11}$ & 0.193 & 0.137 & -0.088 & 0.348 & 0.217 & 0.133 & 0.075 & 0.359 & 0.104 & 0.306 & -0.275 & 0.180 & 0.167 & 0.154 & -0.096 & 0.350 \\
\hline$\theta_{12}$ & -0.454 & 0.094 & 0.121 & 0.370 & .535 & 0.07 & 0.260 & 271 & -0.077 & 383 & -0.067 & 392 & 168 & 0.303 & .068 & 0.390 \\
\hline$\theta_{13}$ & 0.328 & 0.152 & -0.047 & 0.392 & 0.554 & 0.029 & 0.220 & 273 & 0.109 & 0.356 & 0.213 & 0.303 & -0.393 & 0.068 & -0.066 & 0.387 \\
\hline$\theta_{14}$ & -0.054 & 0.312 & 0.012 & 0.394 & -0.160 & 0.053 & -0.064 & 0.290 & -0.055 & 0.299 & -0.114 & 0.167 & 0.184 & 0.014 & 0.008 & 0.397 \\
\hline$\theta_{21}$ & -3.167 & 0.000 & 1.181 & 0.141 & -0.839 & 0.141 & -3.117 & 0.009 & -2.581 & 0.000 & -2.303 & 0.010 & -2.967 & 0.000 & -5.633 & 0.000 \\
\hline$\theta_{22}$ & 0.744 & 0.148 & -0.531 & 0.254 & 0.563 & 0.133 & 0.709 & 0.278 & -0.885 & 0.165 & -0.183 & 0.387 & -0.861 & 0.185 & 3.309 & 0.004 \\
\hline$\theta_{31}$ & -0.064 & 0.303 & $\begin{array}{l}-0.378 \\
\end{array}$ & 0.005 & -0.261 & 0.003 & -0.017 & 0.398 & 0.211 & 0.042 & -0.269 & 0.150 & 0.022 & 0.365 & $\begin{array}{l}-0.798 \\
\end{array}$ & 0.000 \\
\hline$\theta_{32}$ & -0.051 & 0.242 & 0.061 & 0.287 & 0.011 & 0.391 & -0.129 & 0.237 & -0.162 & 0.009 & -0.035 & 0.378 & -0.074 & 0.033 & 0.193 & 0.091 \\
\hline
\end{tabular}

NOTE: ANDA: Andalucia; ARA: Aragon; ASTU: Asturias; BALE: Balearic Islands; CANA: Canary Islands; CANT: Cantabria; CLEO: Castille-Leon; CMAN: Castille-La Mancha; CATA: Catalonia; CVAL: Valencian Community; EXTR: Extremadura; GALI: Galicia; MADR: Community of Madrid; NAVA: Navarre;

PAVA: Basque Country; RIOJ: La Rioja 


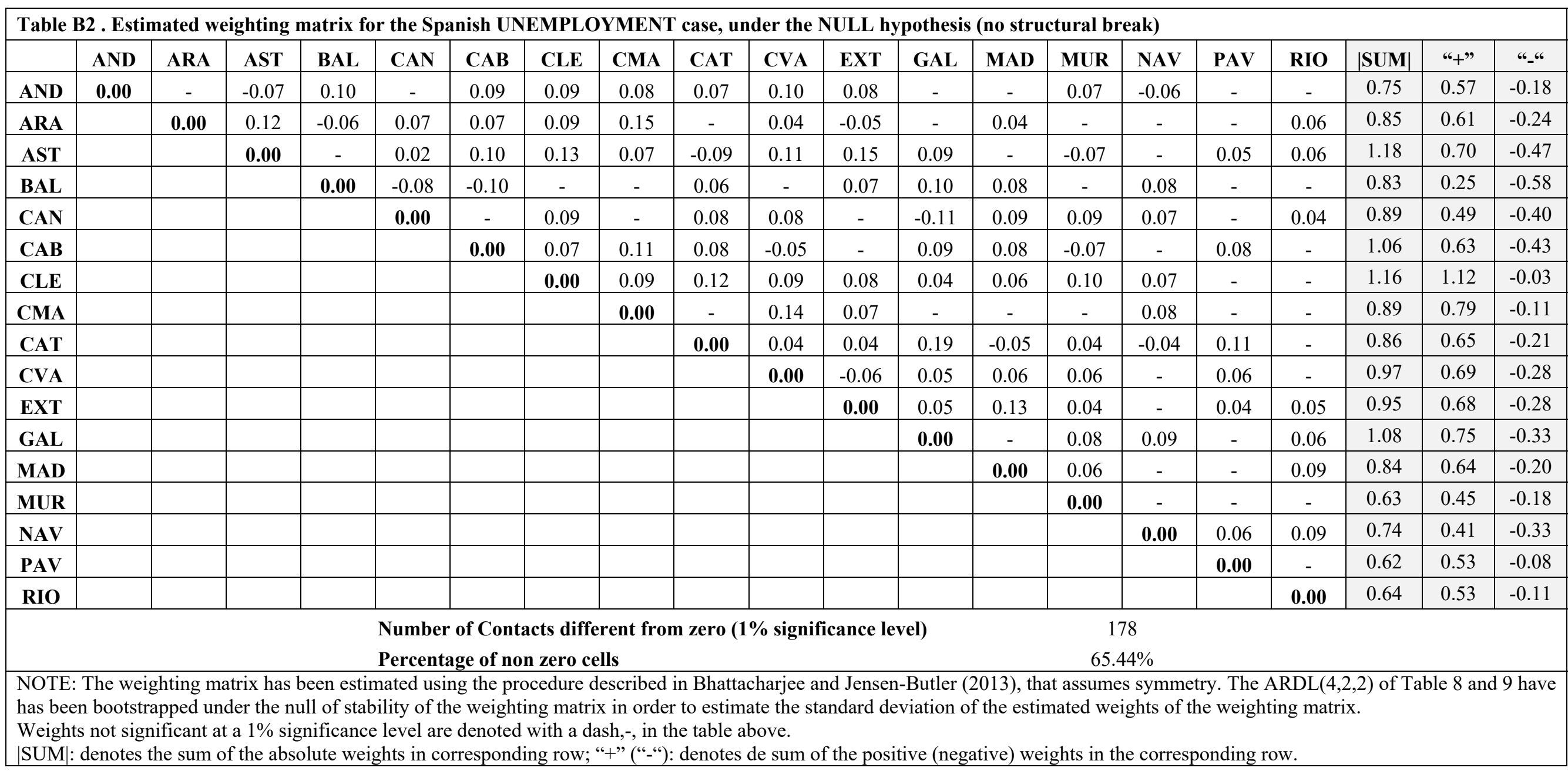




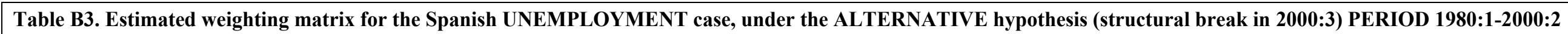

\begin{tabular}{|c|c|c|c|c|c|c|c|c|c|c|c|c|c|c|c|c|c|c|c|c|}
\hline & AND & ARA & AST & BAL & CAN & CAB & CLE & CMA & CAT & CVA & EXT & GAL & MAD & MUR & NAV & PAV & RIO & $|\mathbf{S U M}|$ & "+" & "__6 \\
\hline AND & 0.00 & - & - & 0.15 & - & 0.11 & 0.15 & 0.05 & - & - & 0.06 & -0.08 & - & 0.10 & -0.12 & - & - & 1.08 & 0.68 & 0.40 \\
\hline ARA & & 0.00 & 0.10 & -0.11 & - & 0.09 & 0.11 & 0.18 & - & - & -0.09 & - & - & - & 0.11 & - & 0.07 & 1.01 & 0.59 & 0.42 \\
\hline AST & & & 0.00 & - & - & - & 0.14 & 0.07 & - & 0.07 & 0.15 & - & 0.17 & - & - & 0.12 & - & 1.05 & 0.86 & 0.18 \\
\hline BAL & & & & 0.00 & -0.11 & - & - & - & - & - & - & 0.13 & - & - & 0.11 & - & 0.04 & 1.04 & 0.27 & 0.77 \\
\hline CAN & & & & & 0.00 & - & 0.11 & - & - & 0.09 & - & - & 0.09 & 0.08 & 0.06 & 0.08 & - & 0.88 & 0.57 & 0.31 \\
\hline CAB & & & & & & $\mathbf{0 . 0 0}$ & - & - & - & - & 0.08 & 0.10 & 0.10 & -0.13 & -0.09 & 0.09 & 0.08 & 1.14 & 0.58 & 0.57 \\
\hline CLE & & & & & & & 0.00 & - & - & 0.06 & - & - & - & 0.08 & 0.08 & - & 0.08 & 1.09 & 1.05 & 0.04 \\
\hline CMA & & & & & & & & 0.00 & - & 0.19 & 0.13 & - & - & - & - & - & - & 0.89 & 0.70 & 0.19 \\
\hline CAT & & & & & & & & & 0.00 & 0.07 & - & 0.09 & - & - & - & 0.11 & - & 0.65 & 0.51 & 0.14 \\
\hline CVA & & & & & & & & & & 0.00 & - & 0.08 & - & 0.12 & - & - & - & 0.96 & 0.77 & 0.19 \\
\hline EXT & & & & & & & & & & & 0.00 & - & 0.15 & - & - & - & 0.12 & 1.00 & 0.64 & 0.36 \\
\hline GAL & & & & & & & & & & & & 0.00 & - & 0.08 & 0.10 & - & 0.07 & 0.93 & 0.61 & 0.32 \\
\hline MAD & & & & & & & & & & & & & 0.00 & 0.06 & - & - & - & 0.93 & 0.79 & 0.13 \\
\hline MUR & & & & & & & & & & & & & & 0.00 & - & - & - & 0.77 & 0.38 & 0.39 \\
\hline NAV & & & & & & & & & & & & & & & 0.00 & 0.09 & - & 0.86 & 0.36 & 0.50 \\
\hline PAV & & & & & & & & & & & & & & & & 0.00 & - & 0.82 & 0.61 & 0.21 \\
\hline RIO & & & & & & & & & & & & & & & & & 0.00 & 0.70 & 0.52 & 0.18 \\
\hline \multicolumn{21}{|c|}{ Number of Contacts different from zero ( $1 \%$ significance level) } \\
\hline & & & & & \multicolumn{9}{|c|}{ Percentage of non zero cells } & \multicolumn{2}{|c|}{$41.91 \%$} & & & & & \\
\hline
\end{tabular}

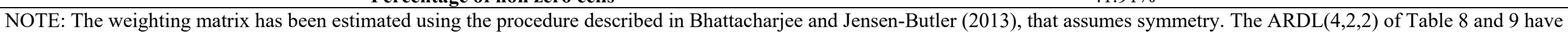
has been bootstrapped under the null of stability of the weighting matrix in order to estimate the standard deviation of the estimated weights of the weighting matrix.

Weights not significant at a $1 \%$ significance level are denoted with a dash,-, in the table above.

$\mid$ SUM|: denotes the sum of the absolute weights in corresponding row; "+" ("“-"): denotes de sum of the positive (negative) weights in the corresponding row. 


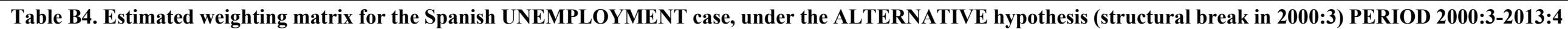

\begin{tabular}{|c|c|c|c|c|c|c|c|c|c|c|c|c|c|c|c|c|c|c|c|c|}
\hline & AND & ARA & AST & BAL & CAN & CAB & CLE & СМА & CAT & CVA & EXT & GAL & MAD & MUR & NAV & PAV & RIO & $|\mathbf{S U M}|$ & “+” & 66_66 \\
\hline AND & 0.00 & - & -0.16 & - & -0.04 & 0.06 & - & 0.10 & - & 0.22 & 0.08 & 0.09 & -0.11 & 0.07 & - & - & - & 0.80 & 0.44 & 0.37 \\
\hline ARA & & $\mathbf{0 . 0 0}$ & 0.15 & - & 0.12 & 0.11 & - & 0.17 & - & 0.06 & - & - & - & - & -0.09 & - & 0.07 & 0.92 & 0.72 & 0.19 \\
\hline AST & & & 0.00 & - & 0.04 & 0.16 & 0.15 & 0.06 & -0.20 & 0.17 & 0.20 & 0.19 & -0.13 & -0.16 & - & - & 0.15 & 2.01 & 0.57 & 1.44 \\
\hline BAL & & & & 0.00 & -0.04 & -0.10 & - & - & -0.01 & - & 0.16 & 0.08 & - & - & - & - & 0.07 & 0.70 & 0.28 & 0.43 \\
\hline CAN & & & & & 0.00 & - & 0.05 & - & 0.17 & 0.05 & -0.05 & -0.17 & 0.09 & 0.10 & 0.15 & - & - & 1.18 & 0.40 & 0.78 \\
\hline CAB & & & & & & 0.00 & 0.09 & 0.09 & 0.15 & -0.10 & -0.11 & 0.05 & 0.09 & - & 0.12 & 0.09 & -0.05 & 1.41 & 0.64 & 0.77 \\
\hline CLE & & & & & & & 0.00 & 0.15 & 0.19 & 0.15 & 0.10 & - & 0.09 & 0.12 & 0.08 & - & -0.11 & 1.35 & 1.14 & 0.22 \\
\hline CMA & & & & & & & & 0.00 & - & 0.06 & - & - & - & - & 0.16 & -0.05 & - & 1.07 & 0.80 & 0.27 \\
\hline CAT & & & & & & & & & 0.00 & 0.08 & 0.15 & 0.28 & -0.13 & 0.07 & -0.13 & 0.09 & - & 1.37 & 0.82 & 0.55 \\
\hline CVA & & & & & & & & & & 0.00 & -0.12 & - & 0.13 & - & -0.07 & 0.04 & - & 1.35 & 0.73 & 0.62 \\
\hline EXT & & & & & & & & & & & 0.00 & 0.08 & 0.13 & 0.09 & - & - & -0.05 & 1.43 & 0.73 & 0.70 \\
\hline GAL & & & & & & & & & & & & 0.00 & - & 0.08 & - & 0.10 & 0.06 & 1.30 & 0.93 & 0.37 \\
\hline MAD & & & & & & & & & & & & & 0.00 & 0.06 & - & - & 0.13 & 1.01 & 0.51 & 0.50 \\
\hline MUR & & & & & & & & & & & & & & 0.00 & - & - & 0.10 & 0.67 & 0.50 & 0.17 \\
\hline NAV & & & & & & & & & & & & & & & 0.00 & 0.05 & 0.21 & 1.32 & 0.65 & 0.67 \\
\hline PAV & & & & & & & & & & & & & & & & 0.00 & - & 0.57 & 0.41 & 0.16 \\
\hline RIO & & & & & & & & & & & & & & & & & 0.00 & 1.11 & 0.59 & 0.52 \\
\hline \multicolumn{21}{|c|}{ Number of Contacts different from zero ( $1 \%$ significance level) } \\
\hline
\end{tabular}

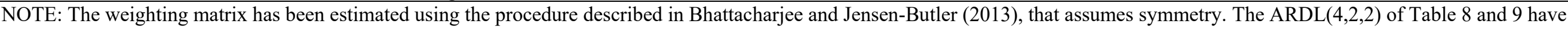
has been bootstrapped under the null of stability of the weighting matrix in order to estimate the standard deviation of the estimated weights of the weighting matrix.

Weights not significant at a $1 \%$ significance level are denoted with a dash,-, in the table above.

$|\mathrm{SUM}|$ : denotes the sum of the absolute weights in corresponding row; "+" ("“-"): denotes de sum of the positive (negative) weights in the corresponding row. 


\section{References}

Aldstadt, J. and A. Getis, 2006, Using AMOEBA to create a spatial weight matrix and identify spatial clusters. Geographical Analysis, 38 327-343.

Angulo, A., P. Burridge and J. Mur, 2016, Testing for a structural break in the weight matrix of the Spatial Error or Spatial Lag model. Working Paper Department of Economic Analysis; University of Zaragoza

Anderson, T., 2003, An Introduction to Multivariate Analysis, 3rd ed. New York: John Wiley \& Sons.

Arellano, M., and S. Bond, 1991, Some tests of specification for panel data: Monte Carlo evidence and an application to employment equations. Review of Economic Studies, 58 277-29.

Ahrens, A. and A. Bhattacharjee, 2015, Two-Step Lasso Estimation of the Spatial Weights Matrix. Econometrics, 3 128-155.

Bai, Z., Jiang, D., Yao, J. and S. Zheng, 2009, Corrections to LRT on largedimensional covariance matrix by RMT. The Annals of Statistics, 37 3822-3840.

Bai, J. and S. Ng, 2010, Panel Unit Root Tests with Cross-Section Dependence: A Further Investigation. Econometric Theory, 26, 1088-1114.

Banerjee, A. and J. Carrión-i-Silvestre, 2015, Cointegration in panel data with structural breaks and cross-sectional dependence. Journal of Applied Econometrics, 30 1-23.

Battacharjee, A. and C. Jensen-Butler, 2013, Estimation of the Spatial Weights Matrix under Structural Constraints. Regional Science and Urban Economics, 43 617-634.

Beenstock, M. and D. Felsenstein, 2012, Nonparametric estimation of the spatial connectivity matrix using spatial panel data. Geographical Analysis, 44 386-397.

Benjanuvatra, S.,2015, QML estimation of the spatial weight matrix in the MR-SAR model. York, DERS University of York Working Paper 
Beyer, R. and F. Smets, 2015), Labour market adjustments in Europe and the US: How different? European Central Bank. Working Paper Series 1767.

Blanchard, O., and Katz, L., 1992, Regional evolutions. Brookings Papers on Economic Activity, 1 1-75.

Brady, R., 2011, Measuring the diffusion of housing prices across space and over time. Journal of Applied Econometrics, 26 213-231.

Brun, J., C. Carrère, P. Gillaumont and J. de Melo, 2005, Has distance died? Evidence from a panel gravity model. The World Bank Economic Review 19, 99-120.

Cameron, G., J. Muellbauer, and A. Murphy, 2006, Was there a British house price bubble? Evidence from a regional panel. Economics Series Working Papers 276, University of Oxford

Can, A., 1990, The Measurement of Neighborhood Dynamics in Urban House Prices. Economic Geography, 66 254-272

Can, A., 1992, Specification and estimation of hedonic housing price models. Regional Science and Urban Economics, 22 453-474,

Case, A., 1991, Spatial Patterns in Household Demand. Econometrica, 59 953-965.

Choi, I., 2002, Combination unit root tests for cross-sectionally correlated panels, in Econometric Theory and Practice: Frontiers of Analysis and Applied Research: Essays in Honor of Peter C. B. Phillips. Cambridge University Press, Cambridge

Corrado, L. and B. Fingleton, 2012, Where is the economics in spatial econometrics? Journal of Regional Science, 52 210-239

DiFonzo, T., 1990, The estimation of M disaggregate time series when contemporaneous and temporal aggregates are known. The Review of Economics and Statistics, 72 178-82.

Druska, V. and W. Horrace, 2004, Generalized Moments Estimation for Spatial Panel Data: Indonesian Rice Farming. American. Journal of. Agricultural. Economics, 86 185-198 
Fernández E, M. Mayor and J. Rodríguez, 2009, Estimating spatial autoregressive models by GME-GCE techniques. International Regional Science Review, 32 148-172.

García, C, 2012, A simple procedure for the comparison of covariance matrices. BMC Evolutionary Biology 12.

Getis A. and J. Aldstadt, 2004, Constructing the spatial weight matrix using a local statistic. Geographical Analysis, 36 90-104

Gil-Serrate, R., J. López-Laborda and J. Mur, 2011, Revenue Autonomy and Regional Growth: An Analysis of the 25-Year Process of Fiscal Decentralisation in Spain. Environment and Planning A, 43 2626-2648.

Griffith, D., 1996, Some Guidelines for Specifying the Geographic Weights Matrix Contained in Spatial Statistical Models. In Practical Handbook of Spatial Statistics, edited by S. Arlinghaus. Boca Raton: CRC.

Harris, R., J. Moffat and V. Kravtsova, 2011, In search of 'W'. Spatial Economic Analysis, 6 249-270.

Holly, S., M. Pesaran and T. Yamagata, 2010, A spatiotemporal model of house prices in the US. Journal of Econometrics, 158 160-173.

Kelejian, H. and I. Prucha, 2007, HAC estimation in a spatial framework. Journal of Econometrics, 140 131-154.

Kelejian, H. and G. Piras, 2014, Estimation of spatial models with endogenous weighting matrices, and an application to a demand model for cigarettes. Regional Science and Urban Economics, 46 140-149.

Kooijman, S., 1976, Some Remarks on the Statistical Analysis of Grids Especially with Respect to Ecology. Annals of Systems Research $\mathbf{5}$.

Ledoit, O. and M. Wolf, 2002, Some hypothesis tests for the covariance matrix when the dimension is large compared to the sample size. The Annals of Statistics, 30 1081-1102.

Lee, L-f. and J. Yu, 2012, QML Estimation of Spatial Dynamic Panel Data Models with Time Varying Spatial Weights Matrices, Spatial Economic Analysis, 7 31-74. 
Li J. and S. Chen, 2012, Two Sample test for high-dimensional covariance matrices. The Annals of Statistics, 40 908-940.

López-Bazo, E. T. del Barrio. and M. Artis, 2005, The geographical distribution of unemployment in Spain. Regional Studies, 39 305-318.

Marston, S., 1985, Two Views of the Geographic Distribution of Unemployment. Quarterly Journal of Economics 100: 57-69.

Martínez, J. and L. Maza, 2003, Analysis of house prices in Spain. Bank of Spain Working Paper Series 0307.

Meen, G., 1996, Spatial aggregation, spatial dependence and predictability in the UK housing market. Housing Studies, 11 345-372.

Moon, H. and B. Perron, 2004, Testing for a unit root in panels with dynamic factors. Journal of Econometrics, 122 81-126,

Mur, J. and J. Paelinck, 2010, Deriving the W-matrix via p-median complete correlation analysis of residuals. Annals of Regional Science, $\mathbf{4 7}$ 253-267

Oud, J. and H. Folmer, 2008, A Structural Equation Approach to Models with Spatial Dependence. Geographical Analysis, 40 152-166.

Paci, R. and S. Usai, 2009, Knowledge flows across European regions. The Annals of Regional Science, 43 669-690.

Patridge M. and D. Rickman, 1997, The Dispersion of US State Unemployment Rates: The Role of Market and Non-market Equilibrium Factors. Regional Studies 31 503-606.

Pedroni, P., 1999, Critical values for cointegration tests in heterogeneous panels with multiple regressors. Oxford Bulletin of Economics and Statistics, 61, 653-670.

Pedroni, P., 2001, Purchasing Power Parity Tests in Cointegrated Panels. Review of Economics and Statistics, 83 727-731.

Pesaran, M. H., 2004, General diagnostic tests for cross section dependence in panels. University of Cambridge, Faculty of Economics, Cambridge Working Papers in Economics No. 0435. 
Pesaran, M., 2007, A simple panel unit root test in the presence of cross section dependence. Journal of Applied Econometrics 22, 265-312.

Pesaran, M. and R. P. Smith, 1995, Estimating long-run relationships from dynamic heterogeneous panels. Journal of Econometrics, 68 79-113.

Pesaran, M., Y. Shin, and R. P. Smith, 1999, Pooled mean group estimation of dynamic heterogeneous panels. Journal of the American Statistical Association, 94 621-634.

Pesaran, M., Y. Shin and R. Smith, 2001, Bounds testing approaches to the analysis of level relationships. Journal of Applied Econometrics 16, 289-326.

Qu, X. and L-f. Lee, 2015, Estimating a spatial autoregressive model with an endogenous spatial weight matrix. Journal of Econometrics, 184 209-232.

Schott, J., 2007, A test for the equality of covariance matrices when the dimension is large relative to the sample size. Computational Statistics \& Data Analysis 51 6535-6542.

Srivastava, M., 2007, Multivariate theory for analyzing high-dimensional data. Journal of Japan Statistical Society 37 53-86.

Srivastava, M. and H. Yanagihara, 2010, Testing the equality of several covariance matrices with fewer observations than the dimension. Journal of Multivariate Analysis, 101 1319-1329.

Srivastava, M., H. Yanagihara and T. Kubokawa, 2014, Tests for covariance matrices in high dimension with less sample size. Journal of Multivariate Analysis, 130 289-309.

Westerlund, J., 2007, Testing for error correction in panel data. Oxford Bulletin of Economics and Statistics. 69 709-748.

Zeilstra, J. and P. Elhorst, 2014, Integrated Analysis of Regional and National Unemployment Differentials in the European Union. Regional Studies, 48 1739-1755. 